\title{
A RATIONAL SURGERY FORMULA FOR THE LMO INVARIANT
}

\author{
DROR BAR-NATAN AND RUTH LAWRENCE
}

\begin{abstract}
We write a formula for the LMO invariant of a rational homology sphere presented as a rational surgery on a link in $S^{3}$. Our main tool is a careful use of the Århus integral and the (now proven) "Wheels" and "Wheeling" conjectures of B-N, Garoufalidis, Rozansky and Thurston. As steps, side benefits and asides we give explicit formulas for the values of the Kontsevich integral on the Hopf link and on Hopf chains, and for the LMO invariant of lens spaces and Seifert fibered spaces. We find that the LMO invariant does not separate lens spaces, is far from separating general Seifert fibered spaces, but does separate Seifert fibered spaces which are integral homology spheres.
\end{abstract}

\section{Contents}

1. Introduction

1.1. The main result: a rational surgery formula for the LMO invariant

1.2. Plan of the proof

1.3. Plan of the paper

1.4. Acknowledgement

2. Preliminaries

2.1. Wheels and Wheeling

2.2. Formal Gaussian integration

2.3. Surgery, continued fractions and matrices

3. Some Lemmas and preliminary computations 10

\begin{tabular}{lll}
\hline 3.1. & Simple diagram manipulations & 10
\end{tabular}

\begin{tabular}{lll}
\hline 3.2. & Properties of $\Omega$ & 11
\end{tabular}

3.3. Properties of $Z$ M 12

\begin{tabular}{llll}
\hline $3.4 . \quad$ One specific integral & 14
\end{tabular}

3.5. The framed unknot and an alternative formula for $\hat{Z}^{\mathrm{LMO}} \quad 14$

\begin{tabular}{lll}
\hline 4. & The Rational Surgery Formula & 15
\end{tabular}

\begin{tabular}{|ll}
\hline 4.1. & Proof modulo computations
\end{tabular}

4.2. The Hopf link and the open Hopf link 16

\begin{tabular}{lll}
\hline 4.3. & The Hopf chain and the shackling element & 17
\end{tabular}

\begin{tabular}{lll}
\hline 4.4. & The Kontsevich integral of rationally framed links & 18
\end{tabular}

\begin{tabular}{ll}
\hline 5. Some Computations & 19
\end{tabular}

\begin{tabular}{lll}
\hline $5.1 . \quad$ General $(p, q)$ lens spaces & 19
\end{tabular}

$\begin{array}{lll}5.2 . & \text { Seifert fibered spaces with a spherical base } & 20\end{array}$

$\begin{array}{lll}5.3 . & \text { Some } s l(2) \text { computations } & 21\end{array}$

5.4. The LMO invariant of integral homology Seifert fibered spaces 23

\begin{tabular}{ll}
\hline References & 23
\end{tabular}

Date: This edition: Jul. 7, 2000; First edition: May 15, 2000; Our label: RationalSurgery.

This paper is available electronically from http://www.ma.huji.ac.il/ drorbn/ and from http://arXiv.org/abs/math.GT/0007045. 


\section{INTRODUCTION}

In their paper "Wheels, Wheeling, and the Kontsevich Integral of the Unknot", B-N, S. Garoufalidis, L. Rozansky and D. P. Thurston [BGRT] made two conjectures; the "Wheels" conjecture about the value of the Kontsevich integral of the unknot, and the "Wheeling" conjecture about the relationship between the two natural products on the space of uni-trivalent diagrams. We quote here a paragraph from [BGRT], explaining a part of their motivation:

If [the Wheeling conjecture] is true, one would be able to use it along with [the Wheels conjecture] and known properties of the Kontsevich integral (such as its behavior under the operations of change of framing, connected sum, and taking the parallel of a component as in [LM2]) to get explicit formulas for the Kontsevich integral of several [...] knots and links. .. . Likewise, using [these conjectures] and the hitherto known or conjectured values of the Kontsevich integral, one would be able to compute some values of the LMO 3-manifold invariant [LMO], using the "Arhus integral" formula of $[\AA-I-I I I]$.

The "Wheels" and "Wheeling" conjectures are now theorems [Ko, Moc, HV, BLT, Th], and this seems a good time to proceed with the plan outlined above. Thus the purpose of our note is to use Wheels and Wheeling and the Arhus integral to obtain some explicit formulas for the values of the Kontsevich integral and the LMO invariant on various simple knots, links and 3-manifolds, as well as a general formula for the behavior of the LMO invariant under rational surgery over links (previously such formulas existed only for integral surgery).

1.1. The main result: a rational surgery formula for the LMO invariant. The LMO invariant $\hat{Z}^{\mathrm{LMO}}(M)$ of a rational homology 3 -sphere $M$, which is given by surgery on some regular integrally framed link $L$ on $S^{3}$ (we write, $M=S_{L}^{3}$; "regular" means that the linking matrix of $L$ is non-singular), is a properly normalized version of the integral of a certain renormalized version of the Kontsevich integral $Z(L)$ of $L$. Thus following [ $\AA$-I-III], we write (see alternative form at Equation (22))

$$
\begin{array}{rlrl}
\check{Z}(L) & :=\nu^{\otimes X} Z(L) \in \mathcal{A}\left(\circlearrowleft_{X}\right), & & \\
\AA_{0}(L) & :=\int \sigma \check{Z}(L) d X ; & & Z_{ \pm}:=\AA_{0}\left(\circlearrowleft^{ \pm 1}\right) \\
\hat{Z}^{\mathrm{LMO}}(M) & :=Z_{+}^{-\varsigma_{+}(L)} Z_{-}^{-\varsigma_{-}(L)} \AA_{0}(L) . &
\end{array}
$$

In these equations:

- $X=\left(x_{i}\right)$ is the set of components of $L, \mathcal{A}\left(\circlearrowleft_{X}\right)$ is the space of chord diagrams skeleton consists of $|X|$ directed circles colored by the elements of $X$ modulo the usual $4 T$, STU, AS and IHX relations, but not divided by the framing independence relation. For general background about chord diagrams and related topological and Lie algebraic issues see $[\mathrm{B}-\mathrm{N}]$.

- $\nu=Z(\circlearrowleft) \in \mathcal{A}(\circlearrowleft) \cong \mathcal{A}(\uparrow)$ is the Kontsevich integral of the zero-framed unknot $\circlearrowleft$, regarded as an element of the space $\mathcal{A}(\circlearrowleft) \cong \mathcal{A}(\uparrow)$ of chord diagrams whose skeleton is a single directed circle (modulo the same relations as above), or a single directed line (modulo the same).

\footnotetext{
1 Throughout this paper all the spaces of diagrams that we will consider are graded and we will automatically complete them with respect to the grading, so as to allow infinite sums of terms of increasing degree.
} 
- $\nu^{\otimes X}$ is the $|X|^{\prime}$ th tensor power of $\nu$, regarded as an element of $\mathcal{A}(\uparrow)^{\otimes X}$, the $X^{\prime}$ 'th tensor power of $\mathcal{A}(\uparrow)$. It acts on the Kontsevich integral $Z(L)$ of $L$ using the usual "stick in anywhere" action $\mathcal{A}(\uparrow)^{\otimes X} \otimes \mathcal{A}\left(\circlearrowleft_{X}\right) \rightarrow \mathcal{A}\left(\circlearrowleft_{X}\right)$.

- $\sigma: \mathcal{A}\left(\circlearrowleft_{X}\right) \rightarrow \mathcal{A}\left(\circledast_{X}\right)$ is the formal PBW linear isomorphism between the space $\mathcal{A}\left(\circlearrowleft_{X}\right)$ and the space $\mathcal{A}\left(\circledast_{X}\right)$ (denoted $\mathcal{B}^{\text {links }}(X)$ in $\mathrm{A}-\mathrm{II}$ ) of $X$-marked uni-trivalent diagrams modulo AS, IHX and $X$-flavored link relations (see [A-1], Section 5.2]). The map $\sigma$ is most easily defined as the inverse of the symmetrization map $\chi: \mathcal{A}\left(\circledast_{X}\right) \rightarrow \mathcal{A}\left(\circlearrowleft_{X}\right)$. If $X$ is a singleton, we often suppress it and write $\mathcal{A}(\circledast), \mathcal{A}(\circlearrowleft), \sigma: \mathcal{A}(\circlearrowleft) \rightarrow \mathcal{A}(\circledast)$, etc. We note that $\sigma$ is a homonymous variant of a better known isomorphism $\sigma: \mathcal{A}\left(\uparrow_{X}\right) \rightarrow$ $\mathcal{A}\left(*_{X}\right)$, where $\mathcal{A}\left(\uparrow_{X}\right)$ is the same as $\mathcal{A}\left(\circlearrowleft_{X}\right)$ but with the directed circle skeleton components replaced by directed lines and $\mathcal{A}\left(*_{X}\right)$ is the standard space of $X$-marked unitrivalent diagrams modulo AS and IHX, not reduced by link relations.

- $\int$ is the key ingredient of "formal integration". It can be either the LMO-style "negative dimensional integral" $\int^{(m)}$, or, in the case when $M$ is a rational homology sphere, the "formal Gaussian integration" $\int^{F G}$ of $[\AA-\mathrm{I}-\mathrm{III}]$. (The equality of these two integration theories is in $\mathrm{A}-\mathrm{III}$; a sticky leftover from $[\AA-\mathrm{I}-\mathrm{III}]$, that $\int^{F G}$ is well defined modulo link relations, is our Proposition 2.2). We note that $\int$ is valued in $\mathcal{A}(\emptyset)$, the space of trivalent diagrams modulo AS and IHX (that is, unitrivalent diagrams with no univalent vertices), and that $\mathcal{A}(\emptyset)$ is a commutative algebra under disjoint union.

- $\circlearrowleft^{ \pm 1}$ is the \pm 1 -framed unknot, and $\varsigma_{+}(L)$ and $\varsigma_{-}(L)$ are respectively, the numbers of positive and negative eigenvalues of the covariance matrix of $Z(L)$, which is the linking matrix of $L$ (see [A-1, Definition 2.8 and Claim 1.10]).

It is rather easy to show (see Section 4.1) that if a rational homology 3-sphere $M$ is given by surgery on some rationally framed link $L$, then its LMO invariant $\hat{Z}^{\mathrm{LMO}}(M)$ can be computed using exactly the same formulas (11)-(3), only replacing the input $Z(L)$ (which is not defined for rationally framed links) by some extension thereof, which we will also denote by $Z(L)$. Our main result in this paper is a precise formula for this "rationally-framed Kontsevich integral". We start with some definitions.

Definition 1.1. ( $[$ BGRT $])$ Let $\Omega \in \mathcal{A}(*)$ be given by

$$
\Omega=\exp _{\sqcup} \sum_{m=1}^{\infty} b_{2 m} \omega_{2 m},
$$

where the modified Bernoulli numbers $b_{2 m}$ are defined by the power series expansion

$$
\sum_{m=0}^{\infty} b_{2 m} x^{2 m}=\frac{1}{2} \log \frac{\sinh x / 2}{x / 2}
$$

(so that $b_{2}=1 / 48, b_{4}=-1 / 5760$, etc.) and $\omega_{2 m}$ is the $2 m$-wheel, the degree $2 m$ uni-trivalent diagram made of a $2 m$-gon with $2 m$ legs (so that $\omega_{2}=-\circ, \omega_{4}=\mathfrak{\alpha}, \ldots$, with all vertices oriented counterclockwise).

Definition 1.2. ([ $\AA$-I-III] $)$ For any element $A \in \mathcal{A}\left(*_{X}\right)$, define a map

$$
\hat{A}=\partial_{A}: \mathcal{A}\left(*_{X}\right) \longrightarrow \mathcal{A}\left(*_{X}\right)
$$




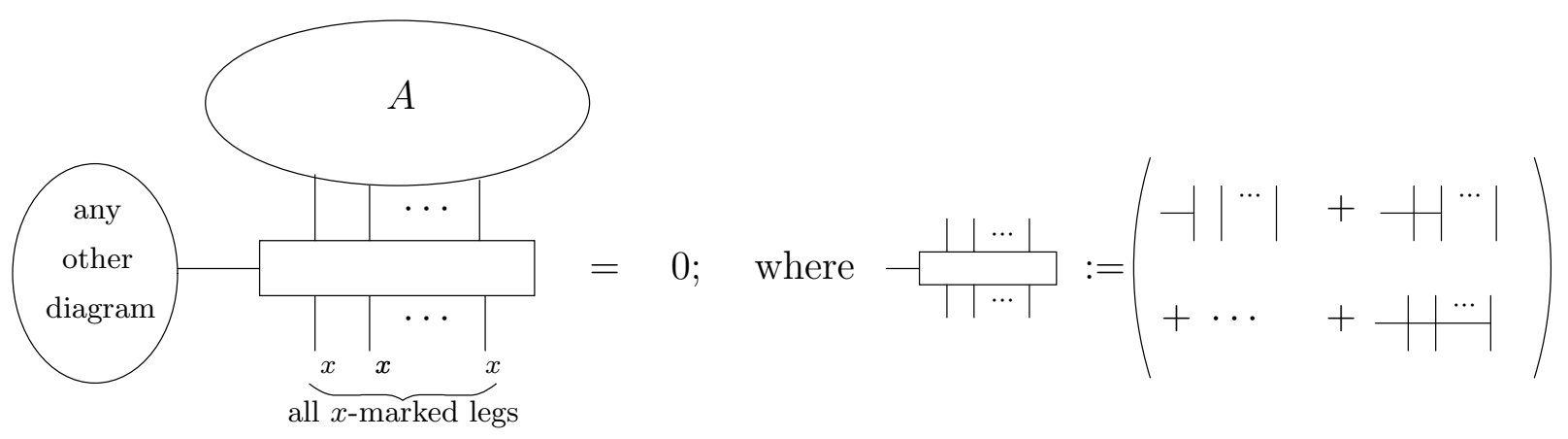

Figure 1. Invariance with respect to $x$ of a diagram $A$.

to act on diagrams $B \in \mathcal{A}\left(*_{X}\right)$ by gluing all legs of $A$ to some subset of legs of $B$ with matching labels. Likewise, define a pairing

$$
\langle A, B\rangle_{X}:=\left(\partial_{A} B\right)_{X \rightarrow 0}:=\left(\begin{array}{l}
\text { sum of all ways of gluing the } x \text {-marked legs } \\
\text { of } A \text { to the } x \text {-marked legs of } B \text {, for all } x \in X
\end{array}\right) .
$$

We note that $\hat{A}=\partial_{A}$ and $\langle A, \cdot\rangle$ are well defined even for arguments $B \in \mathcal{A}\left(\circledast_{X}\right)$, provided $\partial_{A}$ annihilates all $X$-flavored link relations. This is equivalent to saying that $A$ is invariant with respect to $x$ for all $x \in X$, where "invariance" is defined in Figure 1 .

Remark 1.3. Strictly speaking, $\partial_{A} B$ and $\langle A, B\rangle$ are ill-defined if both $A$ and $B$ contain struts (see [A-I], Section 2.2]), so that closed vertex-free loops can be formed by gluing them together. We will not encounter this problem in this paper.

Remark 1.4. Formally $\partial_{A}$ acts as if it were a differential operator on multi-variable polynomials in symbols indexed by $X$, according to an operator obtained from $A$ by replacing each label $x$ by $\partial_{x}$ (see [A-II, Section 2]). Thus while dealing with specific diagrams we will sometimes use notation as follows:

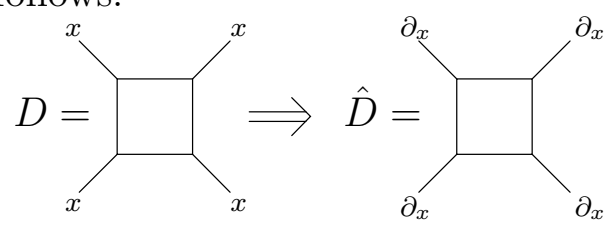

Next we define the Dedekind symbol $S(p / q)$ of a reduced rational number $p / q$. A comprehensive source of information about the Dedekind symbol is [KM], where (among other things) the well-definededness and equivalence of the definitions below is discussed.

Definition 1.5. The Dedekind symbol $S(p / q)$ of a reduced rational number $p / q$ is defined by the properties

$$
S(-x)=-S(x), \quad S(x+1)=S(x) \quad \text { and } \quad S\left(\frac{p}{q}\right)+S\left(\frac{q}{p}\right)=\frac{p}{q}+\frac{q}{p}+\frac{1}{p q}-3 \operatorname{sign}(p q) .
$$

Equivalently, $S(p / q)$ is defined by its relation $S(p / q)=12 \operatorname{sign}(q) s(p, q)$ with the Dedekind sum $s(p, q)$, which is given by either of two formulas

$$
s(p, q):=\sum_{k=1}^{|q|-1}\left(\left(\frac{k}{q}\right)\right)\left(\left(\frac{k p}{q}\right)\right)=\frac{1}{4|q|} \sum_{k=1}^{|q|-1} \cot \left(\frac{k \pi}{q}\right) \cot \left(\frac{k p \pi}{q}\right),
$$


where $((x))$ is the sawtooth function $((x)):=x-\lfloor x\rfloor-1 / 2$. See Table 1 on page 10 .

Definition 1.6. Let $L$ be a rationally framed link, with framing $f_{i}=p_{i} / q_{i}$ on the component $x_{i}$ (measured relative to the 0 framing), and let $L^{0}$ be $L$ with all framings replaced by 0 . Set

$$
Z(L):=Z\left(L^{0}\right) \prod_{i} \chi \hat{\Omega}_{x_{i}}\left(\Omega_{x_{i}}^{-1} \Omega_{x_{i} / q_{i}}\right) \exp \left(\frac{f_{i}}{2} \iota_{x_{i}}+\frac{S\left(f_{i}\right)}{48} \theta\right),
$$

where in this equation:

- $\Omega^{-1}$ refers to inversion with respect to the disjoint union product of $\mathcal{A}(*)$.

- $\Omega_{x}^{-1}$ denotes $\Omega^{-1}$ with all of its univalent vertices ("legs") colored $x$.

- $\Omega_{x / q}$ denotes $\Omega$ with all of its legs colored by $x / q$ (meaning that terms with $k$ legs get multiplied by $\left.1 / q^{k}\right)$.

- $\theta$ denotes the trivalent diagram $\bigcirc$ in $\mathcal{A}(\emptyset)$ and $\iota_{x_{i}}$ denotes the "isolated chord" diagram in $\mathcal{A}\left(\uparrow_{x_{i}}\right)$.

Notice that if all the $f_{i}$ 's are integers, then $\Omega_{x_{i}}^{-1} \Omega_{x_{i} / q_{i}}=1$ and $S\left(f_{i}\right)=0$, and thus $Z(L)=Z\left(L^{0}\right) \prod_{i} e^{f_{i} \iota_{i} / 2}$, and hence the new definition of $Z$ extends the standard definition of the Kontsevich integral of an integrally framed link.

We can finally state our main theorem.

Theorem 1. (Proof on page 10, alternative formulation in Theorem Q). Let L be a rationally framed link and let $M=S_{L}^{3}$ be the rational homology 3-sphere obtained from $S^{3}$ by surgery along L. Then Equations (1)-(3) continue to hold for the computation of $\hat{Z}^{\mathrm{LMO}}(M)$, only using Definition 1.0 for the definition of $Z(L)$.

Remark 1.7. The LMO invariant can be generalized to be an invariant of 3-manifolds with an embedded link, and the surgery formula for the (thus generalized) LMO invariant holds even if the base manifold is not necessarily $S^{3}$. As our proof of Theorem 11 is completely local (see below), the theorem generalizes in the obvious manner to the case when the base manifold is arbitrary and some passive (non-surgery) link is also present.

1.2. Plan of the proof. It is well known (e.g. [Ro, Section $9 \mathrm{H}]$ ) that rational surgery with parameter $p / q$ over a link component can be achieved by shackling that component with a framed Hopf chain and then performing integral surgery, in which the framings $a_{1, \ldots, \ell}$ are related to $p / q$ via a continued fraction expansion (cf. [KM, Equation 0.5]):

(6)

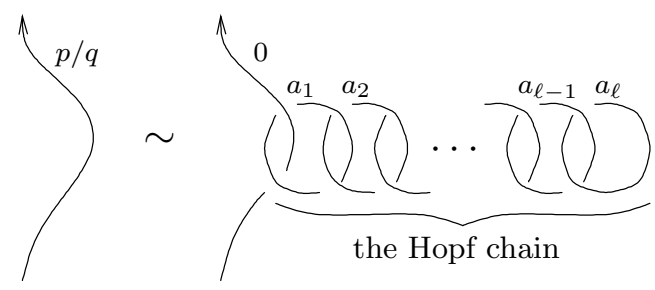

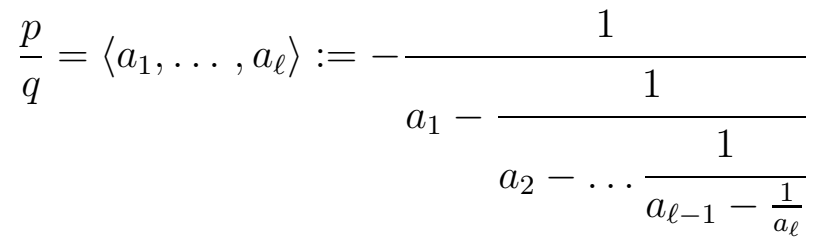

Thus we compute the LMO invariant of $S_{L}^{3}$ by introducing an integrally framed shackled version $L^{s}$ of $L$, in which all of the original components of $L$ are shackled as above, and then by computing $\hat{Z}^{\mathrm{LMO}}\left(S_{L^{s}}^{3}\right)$. We use a lemma, Lemma 4.1 below, that allows us to compute the integral in the definition of $\hat{Z}^{\mathrm{LMO}}\left(S_{L^{s}}^{3}\right)$ in two steps: first we integrate over the Hopf chains, and then over the original components of $L^{0}$. The first step is computational in 
nature, rather complicated in the technical sense and takes up the bulk of the proof. When the output of the first step is appropriately normalized, it turns out to be $Z(L)$, which then needs to be fed into the second step, which is nothing but a re-run of the procedure in Equations (1)-(3). This proves Theorem 11.

1.3. Plan of the paper. In Section 2 we discuss some preliminaries: the Wheels and Wheeling theorems, formal Gaussian integration and assorted facts regarding continued fractions and matrices. In Section 3 we prove some necessary lemmas, and in Section 4 we carry out the computation mentioned above, of the Kontsevich integral and of surgery over Hopf chains, and thus finish the proof of Theorem 1 .

Section 5 contains some further computations. Most importantly, we compute the LMO invariant of arbitrary lens spaces and of certain Seifert fibered spaces, and find that the LMO invariant does not separate lens spaces, is far from separating general Seifert fibered spaces, but it does separate Seifert fibered spaces which are integral homology spheres.

1.4. Acknowledgement. We wish to thank R. Kirby, S. Levy, J. Lieberum, L. Rozansky, H. Rubinstein, D. P. Thurston and B. Weiss for their remarks, suggestions and ideas. In particular we wish to thank D. P. Thurston for his help with the argument in Proposition 2.2. The first author's research at MSRI was supported in part by NSF grant DMS-9701755. The second author's research at the Hebrew University was supported in part by a Guastella Fellowship.

\section{Preliminaries}

2.1. Wheels and Wheeling. Let us start with the statements of the two fundamental theorems, Wheels and Wheeling.

Theorem 2 (Wheels, [BGRT, BLT, Th]). The Kontsevich integral of the unknot, $\nu=Z(\circlearrowleft$ ), is the symmetrization of the Wheels element $\Omega$ of Equation (四): $\nu=\chi \Omega$.

Theorem 3 (Wheeling, [BGRT, [Ko, Mog, BLT, Th]). The map $\chi \circ \hat{\Omega}_{x}: \mathcal{A}\left(*_{x}\right) \longrightarrow \mathcal{A}\left(\uparrow_{x}\right)$ is an algebra isomorphism, so that

$$
\chi \hat{\Omega}_{x}(A \bullet B)=\chi \hat{\Omega}_{x}(A) \# \chi \hat{\Omega}_{x}(B), \quad \text { for } A, B \in \mathcal{A}\left(*_{x}\right) .
$$

We will need a slightly more general form of the Wheeling theorem, also proven in [BLT, Th:

Theorem 3'. Equation $\square$ holds even if $A$ and $B$ are allowed to have skeleton components and other univalent vertices beyond those labeled $x$, provided $A$ and $B$ are both "invariant with respect to $x "$, meaning that they satisfy the relation in Figure 1 .

We note that Theorems 23] have a common generalization as explained and proven in BLT, Th, (see also Section 4.2 of this article):

Theorem 4. Let $\underset{x}{y}$ denote the open Hopf link with the open component labeled $x$ and the closed component labeled $y$. Let $\mathcal{A}\left(\uparrow_{x} \circledast_{y}\right)$ denote the space of diagrams that have one directed interval skeleton component marked $x$ and (possibly) a number of univalent vertices marked 
$y$, modulo the STU, AS and IHX relations and modulo $y$-flavored link relations as in $\mathrm{A}-\mathrm{II}$, Section 5.2]. Then in $\mathcal{A}\left(\uparrow_{x} \circledast y\right)$,

$$
\left.\left.\left.\left.\sigma_{y} Z\left(\stackrel{\leftrightarrow}{x}_{x}^{y}\right)=\Omega_{y} \bullet \exp _{\#}(\lrcorner_{x}^{y}\right)=\left(1+\frac{1}{48} y-\circ y+\ldots\right)\left(\rightarrow_{x}+\right\lrcorner_{x}^{y}+\frac{1}{2}\right\lrcorner_{x}^{y} \#\right\lrcorner_{x}^{y}+\ldots\right)
$$

\subsection{Formal Gaussian integration.}

Definition 2.1. Formal Gaussian integration is defined on formal Gaussian-like expressions ("perturbed $\Lambda$-Gaussians", for some invertible "covariance" $X \times X$ matrix $\Lambda=\left(l_{i j}\right)$ ) in $\mathcal{A}\left(*_{X} E\right)$ ( $E$ denotes some arbitrary extra skeleton components) by

$$
\int^{F G} P \exp (\frac{1}{2} l_{i j} \overbrace{x_{i}}) d X:=\left\langle\exp (-\frac{1}{2} l^{i j} \underbrace{x_{i} x_{j}}), P\right\rangle_{X}=\left.\exp (-\frac{1}{2} l^{i j} \underbrace{\partial_{x_{j}}}_{x_{i}}) P\right|_{X \rightarrow 0}
$$

where $\overbrace{i} \widetilde{x}_{j}(\underbrace{\partial_{x_{i}} \partial_{x_{j}}})$ denotes the $x_{i} x_{j}$-colored $\left(\partial_{x_{i}} \partial_{x_{j}}\right.$-colored) strut, where $P$ is $X$-substantial (involves no struts both of whose ends are colored by colors in $X$, see [A-II, Definition 2.7]), and where $\left(l^{i j}\right)$ is the inverse matrix of $\Lambda=l_{i j}$. (Here and below we employ the Einstein summation convention).

Below we will often need to compute the Kontsevich integral of links, and formal Gaussian integrals thereof. The Kontsevich integral of a link is valued in a quotient space $\mathcal{A}\left(\circledast_{X}\right)$ of $\mathcal{A}\left(*_{X}\right)$, and thus it is useful to note that formal Gaussian integration is well defined for integrands in $\mathcal{A}\left(\circledast_{X}\right)$ :

Proposition 2.2. Formal Gaussian integration is well defined on $\mathcal{A}\left(\circledast_{X} E\right)$. Specifically, if $Z \in \mathcal{A}\left(\circledast_{X} E\right)$ can be written as a perturbed $\Lambda$-Gaussian in two ways,

$$
Z=P_{1} \exp (\frac{1}{2} l_{i j} \overbrace{x_{i}} x_{j})=P_{2} \exp (\frac{1}{2} l_{i j} \overbrace{x_{i}}^{x_{j}})
$$

where $P_{1,2}$ are $X$-substantial and in $\mathcal{A}\left(*_{X} E\right)$ but the equality holds in $\mathcal{A}\left(\circledast_{X} E\right)$, then

$$
\int^{F G} d X P_{1} \exp (\frac{1}{2} l_{i j} \overbrace{i} x_{j})=\int^{F G} d X P_{2} \exp (\frac{1}{2} l_{i j} \overbrace{x_{i}} \widehat{x}_{j})
$$

in $\mathcal{A}(E)$.

Proof. We argue by induction on $|X|$. If $X$ is empty, so is the statement of the proposition. Otherwise there are two cases.

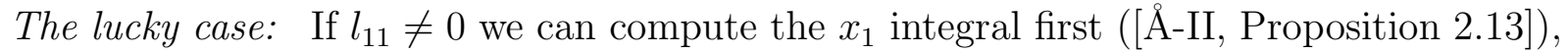
and we need to show that

$$
\int^{F G} d\left(X \backslash\left\{x_{1}\right\}\right) \int^{F G} d x_{1}\left(P_{1}-P_{2}\right) \exp (\frac{1}{2} l_{i j} \overbrace{x_{i}} x_{j})=0,
$$

where we know that $\left(P_{1}-P_{2}\right) \exp (\frac{1}{2} l_{i j} \overbrace{i} \widehat{x}_{j})$ is link relation equivalent to 0 via $X$-flavored link relations. Multiplication by $\overbrace{1} x_{1}$ is well defined modulo link relations (exercise!), and thus

$$
P:=\left(P_{1}-P_{2}\right) \exp (\frac{1}{2} l_{i j} \overbrace{i} \widehat{x}_{j}) \exp (-\frac{1}{2} l_{11} x \overbrace{x_{1}})
$$


is also link relation equivalent to 0 via $X$-flavored link relations. By the definition of formal Gaussian integration the inner integral in Equation (9) is given by

$$
\left\langle P, \exp \left(-\frac{1}{2} l_{11}^{-1} x_{1}^{x_{1} x_{1}}\right)\right\rangle \text {. }
$$

The map $D \mapsto\left\langle D, \exp (-\frac{1}{2} l_{11}^{-1} \underbrace{x_{1} x_{1}})\right\rangle$ kills all $x_{1}$-flavored link relations (because the $x_{1^{-}}$ marked strut $\underbrace{x_{1} x_{1}}$ is $x_{1}$-invariant), and maps $\left(X \backslash\left\{x_{1}\right\}\right)$-flavored link relations to $\left(X \backslash\left\{x_{1}\right\}\right)$ flavored link relations. Therefore the inner integral in Equation (9) is link equivalent to 0 via $\left(X \backslash\left\{x_{1}\right\}\right)$-flavored link relations. By the induction hypothesis we now find that the outer integral in Equation (9) vanishes.

The ugly case: If $l_{11}=0$ we consider

$$
I(\epsilon):=\int^{F G} d X\left(P_{1}-P_{2}\right) \exp (\frac{1}{2} l_{i j} \overbrace{i} x_{j}) \exp \epsilon \overbrace{1} \overbrace{x},
$$

where $\epsilon$ is an arbitrary scalar. Multiplication by $x_{1} \overbrace{x_{1}}$ is well defined modulo link relations, and so the integrand here remains link equivalent to 0 . Thus by the lucky case, $I(\epsilon)$ vanishes for all $\epsilon \neq 0$. On the other hand, the coefficient of every diagram that appears in $I(\epsilon)$ is a rational function in $\epsilon$ which is non singular at $\epsilon=0$ because $\Lambda$ is regular. Thus it must be that $I(0)=0$.

Remark 2.3. The above proof gives us an opportunity to whine and complain about the state of our understanding of integration in spaces of diagrams. Two such integration theories exist. The LMO integration theory as in [LMO, Le], and the formal Gaussian theory of $[\AA$ I-III]. Neither one of them is satisfactory:

- Formal Gaussian integration has a solid conceptual foundation; it is the diagrammatic analogue of an old time favorite, the theory of Gaussian integrals and Feynman diagrams. But it is defined only for a restricted kind of integrands, and thus it can only be used to define an invariant of a restricted class of 3-manifolds, namely rational homology spheres. And certain aspects of it, such as its being well-defined modulo link relations (as above), are somewhat tricky.

- The LMO integration theory is always defined and there's no problem showing that it is well-defined modulo link relations, and thus it is superior to formal Gaussian integration, at least in some sense. But we (the authors) lack a conceptual understanding of what it really means. It involves the introduction out of thin air of some strange relations, and one needs to spend some time verifying that under these new relations the theory does not collapse to nothing. In [LMO, Le], these relations and the construction as a whole are not interpreted. In [A-III] the relations are given a semi-satisfactory interpretation in terms of "negative dimensions". But whereas formal Gaussian integration is clearly the diagrammatic counterpart of Gaussian integration over Lie algebras, the LMO integration theory is not fully understood as the diagrammatic counterpart of anything (be it integration or anything else). The situation clearly needs to be resolved. Is the LMO theory a diagrammatization of something known? What is it? If not, then it is a genuinely new piece of mathematics. Genuinely new mathematics is wonderful, but it is a rare commodity. Is the LMO theory really new? 
In [A-II] it is shown that the two integration theories agree whenever the weaker one is defined. Thus we could have deduced the previous proposition from the corresponding one for the LMO theory, which is easier to prove. But then we would have had to rely on a non trivial theory which is not yet properly understood.

2.3. Surgery, continued fractions and matrices. The continued fraction expansion in Equation (6) has a slightly refined version

$$
\left(\begin{array}{l}
p \\
q
\end{array}\right)=\left(\begin{array}{cc}
0 & -1 \\
1 & 0
\end{array}\right)\left(\begin{array}{cc}
a_{1} & -1 \\
1 & 0
\end{array}\right)\left(\begin{array}{cc}
a_{2} & -1 \\
1 & 0
\end{array}\right) \cdots\left(\begin{array}{cc}
a_{\ell} & -1 \\
1 & 0
\end{array}\right)\left(\begin{array}{l}
1 \\
0
\end{array}\right),
$$

in which the signs of $p$ and $q$ are uniquely determined from $a_{1, \ldots, \ell}$. For convenience and without loss of generality, we assume that the signs of $p$ and $q$ are fixed so that (10) holds. This done, we define the integers $u$ and $v$ from the equality

$$
\left(\begin{array}{ll}
p & u \\
q & v
\end{array}\right)=A\left(a_{1}, \ldots, a_{\ell}\right):=\left(\begin{array}{cc}
0 & -1 \\
1 & 0
\end{array}\right)\left(\begin{array}{cc}
a_{1} & -1 \\
1 & 0
\end{array}\right)\left(\begin{array}{cc}
a_{2} & -1 \\
1 & 0
\end{array}\right) \cdots\left(\begin{array}{cc}
a_{\ell} & -1 \\
1 & 0
\end{array}\right) .
$$

(cf. [KM, Lemma 1.9]).

Let $\Lambda=\left(l_{i j}\right)$ be the (tri-diagonal) linking matrix of the Hopf chain of Equation (6) (cf. [KM], Equation 0.6]),

$$
\Lambda=\Lambda\left(a_{1}, \ldots, a_{\ell}\right):=\left(\begin{array}{ccccc}
a_{1} & 1 & & & \\
1 & a_{2} & 1 & & \\
& 1 & \ddots & & \\
& & & \ddots & 1 \\
& & & 1 & a_{\ell}
\end{array}\right)
$$

Proposition 2.4. The four corners of the inverse matrix $\Lambda^{-1}=\left(l^{i j}\right)$ of $\Lambda$ are given by

$$
l^{11}=-\frac{p}{q}, \quad l^{1 \ell}=l^{\ell 1}=\frac{(-1)^{\ell}}{q}, \quad l^{\ell \ell}=-\frac{v}{q} .
$$

Proof. By induction on $\ell$ (and row expansion of the relevant determinants) one establishes the equality

$$
A\left(a_{1}, \ldots, a_{\ell}\right)=\left(\begin{array}{cc}
-\operatorname{det} \Lambda\left(a_{2}, \ldots, a_{\ell}\right) & \operatorname{det} \Lambda\left(a_{2}, \ldots, a_{\ell-1}\right) \\
\operatorname{det} \Lambda\left(a_{1}, \ldots, a_{\ell}\right) & -\operatorname{det} \Lambda\left(a_{1}, \ldots, a_{\ell-1}\right)
\end{array}\right) .
$$

After that, the theorem follows from simple observations regarding the determinant $\operatorname{det} \Lambda$ and the minors $\Lambda^{(i j)}$ of the matrix $\Lambda$. Namely, that $\operatorname{det} \Lambda=\operatorname{det} \Lambda\left(a_{1}, \ldots, a_{\ell}\right)$, that $\Lambda^{(11)}$ is $\operatorname{det} \Lambda\left(a_{2}, \ldots, a_{\ell}\right)$, that $\Lambda^{(1 \ell)}$ is triangular with ones on the diagonal, and that $\Lambda^{(\ell \ell)}$ is $\operatorname{det} \Lambda\left(a_{1}, \ldots, a_{\ell-1}\right)$.

We note that while the matrix $\Lambda$ is not determined by $p / q$, a certain combination of its trace $\tau$, its signature $\varsigma$ and the numbers $p / q$ and $v / q$ appearing in the corners of its inverse matrix does depend only on $p / q$ :

Theorem 5. (cf. [KM, Equation 0.8]) The Dedekind symbol $S(p / q)$ of $p / q$ (see Table [1) is given by

$$
S(p / q)=3 \varsigma-\tau-l^{11}-l^{\ell \ell}=3 \varsigma-\tau+\frac{p+v}{q} .
$$




\begin{tabular}{|c|c|c|c|c|c|c|c|c|c|c|c|c|}
\hline$p$ & 1 & 2 & 3 & 4 & 5 & 6 & 7 & 8 & 9 & 10 & 11 & 12 \\
\hline$q=1$ & 0 & 0 & 0 & 0 & 0 & 0 & 0 & 0 & 0 & 0 & 0 & 0 \\
\hline$q=2$ & 0 & . & 0 & . & 0 & . & 0 & . & 0 & 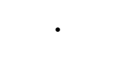 & 0 & $\cdot$ \\
\hline$q=3$ & 2 & -2 & . & 2 & -2 & . & 2 & -2 & . & 2 & -2 & 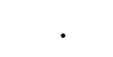 \\
\hline$q=4$ & 6 & . & -6 & . & 6 & . & -6 & . & 6 & . & -6 & 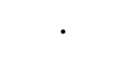 \\
\hline$q=5$ & 12 & 0 & 0 & -12 & . & 12 & 0 & 0 & -12 & . & 12 & 0 \\
\hline$q=6$ & 20 & . & . & . & -20 & . & 20 & . & . & . & -20 & . \\
\hline$q=7$ & 30 & 6 & -6 & 6 & -6 & -30 & . & 30 & 6 & -6 & 6 & -6 \\
\hline$q=8$ & 42 & . & 6 & . & -6 & . & -42 & . & 42 & . & 6 & . \\
\hline$q=9$ & 56 & 16 & . & -16 & 16 & . & -16 & -56 & . & 56 & 16 & . \\
\hline$q=10$ & 72 & . & 0 & . & . & . & 0 & . & -72 & . & 72 & . \\
\hline$q=11$ & 90 & 30 & 18 & 18 & -30 & 30 & -18 & -18 & -30 & -90 & . & 90 \\
\hline$q=12$ & 110 & . & . & . & -2 & . & 2 & . & . & . & -110 & 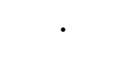 \\
\hline$q=13$ & 132 & 48 & 12 & -12 & 0 & -48 & 48 & 0 & 12 & -12 & -48 & -13 \\
\hline$q=14$ & 156 & . & 36 & . & 36 & . & . & . & -36 & . & -36 & . \\
\hline$q=15$ & 182 & 70 & . & 38 & . & . & -70 & 70 & . & . & -38 & . \\
\hline$q=16$ & 210 & . & 30 & . & -30 & . & -18 & . & 18 & . & 30 & 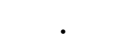 \\
\hline$q=17$ & 240 & 96 & 60 & 0 & 12 & 60 & 12 & -96 & 96 & -12 & -60 & -12 \\
\hline$q=18$ & 272 & . & . & . & 16 & . & -16 & . & . & . & 16 & . \\
\hline$q=19$ & 306 & 126 & 54 & 66 & 66 & -54 & 18 & -18 & -126 & 126 & 18 & -18 \\
\hline$q=20$ & 342 & . & 90 & . & . & . & 90 & . & -42 & & 42 & . \\
\hline$q=21$ & 380 & 160 & . & 20 & -20 & . & 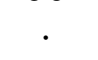 & 16 & . & -160 & 160 & . \\
\hline$q=22$ & 420 & . & 84 & . & 36 & . & -84 & . & 36 & . & . & . \\
\hline$q=23$ & 462 & 198 & 126 & 102 & 42 & 102 & -6 & 126 & -42 & -6 & -198 & 198 \\
\hline$=24$ & 506 & . & . & . & 106 & . & 38 & . & . & . & -74 & . \\
\hline$q=25$ & 552 & 240 & 120 & 48 & . & -48 & 0 & -120 & 48 & 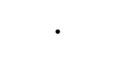 & -48 & -240 \\
\hline
\end{tabular}

Table 1. Some values of $q \cdot S(p / q)$ for relatively prime $p, q$. Recall that $S$ is antisymmetric and periodic with period 1 , and hence all values of $S(p / q)$ with $q \leq 25$ can be recovered from this table. (Change the first line of RationalSurgery. tex to $\backslash \operatorname{def} \backslash \operatorname{STable}\{I\}$ to print a denser table that runs up to $q=65)$.

\section{Some LEMmas AND PRELIMINARY COMPUTATIONS}

3.1. Simple diagram manipulations. In the previous section we have used elements of $\mathcal{A}\left(*_{X}\right)$ where $X$ is a set of labels. One may extend this notation in a multilinear way so as to allow labels which are elements of a formal vector space whose basis is indexed by $X$. Thinking of the elements of $\mathcal{A}\left(*_{X}\right)$ as multinomials in the symbols $X$, whose coefficients are diagrams, one can also perform evaluations in which one or more of the symbols in $X$ are set to zero, which has the effect of selecting out those diagrams not mentioning these symbols.

Lemma 3.1. For all $A, B \in \mathcal{A}\left(*_{X}\right), \partial_{A}(B)=\left\langle A_{\bar{X}}, B_{X+\bar{X}}\right\rangle_{\bar{X}}$. Here $\bar{X}$ denotes a set in 1-1 correspondence to $X, X+\bar{X}$ is the set of corresponding sums, $A_{\bar{X}}$ denotes the same diagram as $A$ with all labels changed from $X$ labels to their corresponding $\bar{X}$-label, while the pairing takes place over the set of labels $\bar{X}$. 
Lemma 3.2. Let $E$ be some skeleton specification (a list of colored $\uparrow$ 's, $\circlearrowleft ' s, *$ 's and $\circledast$ 's) not containing the colors $t, x$ and $y$. For any $D \in \mathcal{A}\left(*_{t}\right)$ and $A \in \mathcal{A}\left(*_{t} E\right)$,

$$
\left[\hat{D}_{t} A\right]_{t \rightarrow x+y}=\hat{D}_{x}[A]_{t \rightarrow x+y}=\hat{D}_{y}[A]_{t \rightarrow x+y} .
$$

\subsection{Properties of $\Omega$.}

Proposition 3.3 (Pseudo-linearity of $\log \Omega$ ). For any $x$-invariant diagram $D$,

$$
\partial_{D} \Omega_{x}=\left.\partial_{D} \Omega_{x}\right|_{x=0} \Omega_{x}=\left\langle D, \Omega_{x}\right\rangle_{x} \Omega_{x}
$$

(compare with standard calculus: if $D$ is any differential operator and $f$ is a linear function, then $D e^{f}=(D f)(0) e^{f}$; we added the prefix "pseudo" above because Equation 19 does not hold for every $D$, but only for $x$-invariant $D$ 's).

Proof. In [BLT, Th] it is shown that $\Omega_{x+y}=\Omega_{x} \bullet \Omega_{y}$ in $\mathcal{A}\left(\circledast_{x} \circledast_{y}\right)$, and thus using Lemma 3.1,

$$
\partial_{D}(\Omega)=\left\langle D_{y}, \Omega_{x+y}\right\rangle_{y}=\left\langle D_{y}, \Omega_{x} \cup \Omega_{y}\right\rangle_{y}=\left\langle D_{y}, \Omega_{y}\right\rangle_{y} \Omega_{x}=\left\langle D_{x}, \Omega_{x}\right\rangle_{x} \Omega_{x} .
$$

The $x$-invariance of $D$ is used in asserting the equality between the contractions $\left\langle D_{y}, \Omega_{x+y}\right\rangle_{y}$ and $\left\langle D_{y}, \Omega_{x} \cup \Omega_{y}\right\rangle_{y}$. If $x$-invariance is not assumed, the contraction map $\left\langle D_{y}, \cdot\right\rangle_{y}: \mathcal{A}\left(*_{x} *_{y}\right) \rightarrow$ $\mathcal{A}\left(*_{x}\right)$ may not descend to a map $\mathcal{A}\left(\circledast_{x} \circledast_{y}\right) \rightarrow \mathcal{A}\left(\circledast_{x}\right)$.

Corollary 3.4. For any $D \in \mathcal{A}(*)$ we have $e^{\partial_{D}} \Omega=e^{\langle D, \Omega\rangle} \Omega$. In particular, for any $\alpha$,

$$
\exp (\frac{\alpha}{2} \underbrace{}_{x} \partial_{x}) \Omega_{x}=\exp \left(\frac{\alpha \theta}{48}\right) \Omega_{x}
$$

Proof. The first assertion follows immediately from Proposition 3.3. The second assertion follows from the first and from the equality $\langle\smile, \Omega\rangle=\theta / 24$, which follows from the fact that the two-legged part of $\Omega$ is $-\circ / 48$.

Corollary 3.5. For any $A, B \in \mathcal{A}(*)$,

$$
\langle A \cup B, \Omega\rangle=\langle A, \Omega\rangle\langle B, \Omega\rangle \text {. }
$$

In particular, if $A$ is invertible, then $\left\langle A^{-1}, \Omega\right\rangle=\langle A, \Omega\rangle^{-1}$.

Proof. Clearly, $\partial_{A \cup B}=\partial_{A} \circ \partial_{B}$. And so, using Proposition 3.3 twice and the fact that $\left.\Omega\right|_{x=0}=1$, we get

$$
\begin{aligned}
\langle A \cup B, \Omega\rangle & =\left.\partial_{A \cup B} \Omega\right|_{x=0}=\left.\partial_{A}\left(\partial_{B}(\Omega)\right)\right|_{x=0} \\
& =\left.\partial_{A}(\Omega)\right|_{x=0} \cdot\langle B, \Omega\rangle=\left.\Omega\right|_{x=0} \cdot\langle A, \Omega\rangle\langle B, \Omega\rangle=\langle A, \Omega\rangle\langle B, \Omega\rangle .
\end{aligned}
$$

Lemma 3.6. The following two formulas hold when observed:

$$
\begin{aligned}
\hat{\Omega} \exp \left(\frac{\frown}{2}\right) & =\exp \left(\frac{\theta}{48}\right) \Omega \exp \left(\frac{\frown}{2}\right) \\
\hat{\Omega}_{x} \exp \left(\frac{x+\overparen{y} x+y}{2}\right) & =\exp \left(\frac{\theta}{48}\right) \Omega_{x+y} \exp \left(\frac{x+\overparen{y} x+y}{2}\right)
\end{aligned}
$$

\footnotetext{
${ }^{2}$ See $[\mathrm{Me}]$.
} 
Proof. Equation (13) follows from the following computation:

$$
\begin{aligned}
& \hat{\Omega}_{x} \exp \left(\frac{\overparen{x} x}{2}\right)=\left\langle\exp \left(\frac{x+\overparen{x}+y}{2}\right), \Omega_{y}\right\rangle_{y} \\
& =\left\langle e^{\widetilde{x x} / 2} e^{\widetilde{x y}} e^{\widetilde{y y} / 2}, \Omega_{y}\right\rangle_{y}=e^{\widetilde{x x} / 2}\left\langle\widetilde{e^{x y}} e^{\widetilde{y y} / 2}, \Omega_{y}\right\rangle_{y} \\
& =e^{\widetilde{x x} / 2}\left\langle e^{\widetilde{x y}}, e^{\partial_{y} \underbrace{\partial_{y}} / 2} \Omega_{y}\right\rangle_{y} \\
& =\exp \left(\frac{\theta}{48}\right) e^{\widetilde{x x} / 2}\left\langle e^{\widetilde{x y}}, \Omega_{y}\right\rangle_{y} \\
& =\exp \left(\frac{\theta}{48}\right) e^{\widetilde{x} / 2} \Omega_{x}
\end{aligned}
$$

The operator $\hat{\Omega}$ commutes with translation by $y$ (the map $x \mapsto x+y$ ), because $\hat{\Omega}$ has "constant coefficients". Thus Equation (14) is a consequence of Equation (13). Alternatively, it can be proven directly along the same lines.

3.3. Properties of $Z^{\natural}$. The Wheeling Theorem allows us to introduce a variant $Z^{\natural}$ of the Kontsevich integral $Z$ which has some nice multiplicative properties:

Definition 3.7. If $L$ is an $X$-marked link (or tangle), set $Z^{\natural}:=\left(\prod_{i} \hat{\Omega}_{x_{i}}^{-1} \circ \sigma_{x_{i}}\right) \circ Z$ to be the "wheeled Kontsevich integral". As $\sigma$ and $\hat{\Omega}^{-1}$ are invertible, $Z^{\propto}$ carries just as much information as the original Kontsevich integral. In a completely parallel manner, set $\check{Z}^{\circ}:=\left(\prod_{i} \hat{\Omega}_{x_{i}}^{-1} \circ \sigma_{x_{i}}\right) \circ \check{Z}$.

A first example of a nice multiplicative property of $Z^{\alpha}$ is the following lemma:

Lemma 3.8. If $L$ is a (possibly rationally) framed link and $L^{0}$ is the corresponding 0 -framed link, then

$$
Z^{\propto}(L)=Z^{\propto}\left(L^{0}\right) \prod_{i} \exp \left(\left(S\left(f_{i}\right)-f_{i}\right) \frac{\theta}{48}\right) \Omega_{x_{i}}^{-1} \Omega_{x_{i} / q_{i}} \exp \frac{f_{i}}{2} \overbrace{x_{i}} \widetilde{x}_{i} .
$$

In particular, if $L$ is integrally framed, then simply

$$
Z^{\alpha}(L)=Z^{\grave{\alpha}}\left(L^{0}\right) \prod_{i} \exp \left(-\frac{f_{i} \theta}{48}\right) \exp \frac{f_{i}}{2} \overbrace{i} \widehat{x}_{i} .
$$

Proof. Indeed,

$$
\begin{aligned}
& Z^{\emptyset}(L)=\left(\prod_{i} \hat{\Omega}_{x_{i}}^{-1} \sigma_{x_{i}}\right) Z(L) \\
& =Z^{\not{\alpha}}\left(L^{0}\right) \prod_{i} \Omega_{x_{i}}^{-1} \Omega_{x_{i} / q_{i}} \exp \left(\frac{f_{i}}{2} \hat{\Omega}_{x_{i}}^{-1} \sigma_{x_{i}}\left\llcorner_{x_{i}}+\frac{S\left(f_{i}\right)}{48} \theta\right) \quad \begin{array}{r}
\text { by Definition } 1.6 \\
\text { and Wheeling }
\end{array}\right. \\
& =Z^{\not}\left(L^{0}\right) \prod_{i} \Omega_{x_{i}}^{-1} \Omega_{x_{i} / q_{i}} \exp \left(\frac{f_{i}}{2}(\overbrace{x_{i}}-\frac{\theta}{24})+\frac{S\left(f_{i}\right)}{48} \theta\right) \quad \begin{array}{r}
\text { as } \sigma \bullet=\frown \text { and as } \\
\Omega=1+\frac{\infty}{48}+\ldots
\end{array}
\end{aligned}
$$


Another nice multiplicative property of $Z^{\ltimes}$ is its behavior under the operation of taking the connected sum of links:

Lemma 3.9. Let $L_{i}$ be an $X_{i}$-marked link and let $x_{i}$ be some specific colors in $X_{i}$, for $i=1,2$, with $X_{i}$ disjoint sets of colors. Let $t$ be a color not in $X_{1} \cup X_{2}$, and let $L$ be the connected sum of $L_{1}$ and $L_{2}$ along the components $x_{1}$ and $x_{2}$, with the "merged" component marked $t$. Then

$$
\begin{aligned}
Z^{\propto}(L) & =Z^{\propto}\left(\circlearrowleft_{t}\right)^{-1}\left(Z^{\propto}\left(L_{1}\right) / x_{1} \rightarrow t\right)\left(Z^{\propto}\left(L_{2}\right) / x_{2} \rightarrow t\right) \\
& =\langle\Omega, \Omega\rangle \Omega_{t}^{-1}\left(Z^{\not}\left(L_{1}\right) / x_{1} \rightarrow t\right)\left(Z^{\propto}\left(L_{2}\right) / x_{2} \rightarrow t\right) .
\end{aligned}
$$

Proof. Equation (15) follows immediately from the generalized form of the Wheeling Theorem, Theorem [3], and the known multiplicative property of the Kontsevich integral $Z$. Equation (16) follows from Equation (15) using the following lemma, which is of independent interest.

Lemma 3.10. The wheeled Kontsevich integral of the 0-framed unknot is given by

$$
Z^{\not}(\circlearrowleft)=\hat{\Omega}^{-1} \sigma \nu=\langle\Omega, \Omega\rangle^{-1} \Omega \text {. }
$$

Proof.

$$
\begin{array}{rlrl}
Z^{\natural}(\circlearrowleft)=\hat{\Omega}^{-1} \sigma \nu & =\hat{\Omega}^{-1} \Omega & \text { by Wheels } \\
& =\left\langle\Omega^{-1}, \Omega\right\rangle \Omega, & & \text { by Proposition } 3.3, \\
& =\langle\Omega, \Omega\rangle^{-1} \Omega, & & \text { by Corollary } 3.5 .
\end{array}
$$

Corollary 3.11. If $L$ is an $X$-marked link, then

$$
\check{Z}^{\propto}(L)=\langle\Omega, \Omega\rangle^{-|X|}\left(\prod_{x \in X} \Omega_{x}\right) Z^{\propto}(L) .
$$

Recall that the integration by parts formula of [A-1], Proposition 2.15] implies that for any $D, Z \in \mathcal{A}\left(*_{x}\right)$ one has $\int \hat{D} Z d x=\int Z d x$ ( $\hat{D}$ is divergence-free $)$. In particular, $\int \check{Z}^{\ltimes} d X=$ $\int \sigma \check{Z} d X$ and thus:

Corollary 3.12. If $L$ is an $X$-marked link (possibly rationally framed), then

$$
\AA_{0}(L)=\langle\Omega, \Omega\rangle^{-|X|} \int\left(\prod_{x \in X} \Omega_{x}\right) Z^{\not{\alpha}}(L) d X .
$$

Exercise 3.13. Determine the behavior of $Z^{\not \alpha}$ under

- doubling a component (see [LM2] and Proposition 4.2 below).

- dropping a component. 
3.4. One specific integral. At several points below we will need the value of a certain specific integral, which we hereby evaluate:

Lemma 3.14. For any scalars $\alpha, \beta$ and $\gamma$ the following equality holds:

$$
I(\alpha, \beta ; \gamma):=\int \Omega_{\alpha x} \Omega_{\beta x} \exp \left(\frac{\gamma}{2} \widetilde{x}\right) d x=\exp \left(-\frac{\alpha^{2}+\beta^{2}}{48 \gamma} \theta\right)\left\langle\Omega_{x}, \Omega_{\alpha \beta x / \gamma}\right\rangle_{x}
$$

Proof.

$$
\begin{aligned}
& I(\alpha, \beta ; \gamma)=\left.\exp (-\frac{1}{2 \gamma} \underbrace{\partial_{u}}_{u}) \Omega_{\alpha u} \Omega_{\beta u}\right|_{u=0} \quad \text { by (8), with a dummy } u \\
& =\left.\exp (-\frac{1}{2 \gamma} \underbrace{\partial^{\prime}}_{\underbrace{}_{u} \partial_{u}}) \Omega_{\alpha(u+v)} \Omega_{\beta(u-v)}\right|_{u=v=0} \quad \text { introducing a spurious } v \\
& =\left.\exp \left(-\frac{1}{2 \gamma}(\alpha \partial_{x}+\underbrace{\left.\beta \partial_{y}\right) \quad\left(\alpha \partial_{x}\right.}+\beta \partial_{y})\right) \Omega_{x} \Omega_{y}\right|_{x=y=0} \quad\left\{\begin{aligned}
\alpha(u+v) & \rightarrow x \\
\beta(u-v) & \rightarrow y \\
\partial_{u} & \rightarrow \alpha \partial_{x}+\beta \partial_{y}
\end{aligned}\right. \\
& =\left.e^{-\frac{\alpha \beta{ }^{\partial x} \partial_{y}}{\gamma}} e^{-\frac{\alpha^{2} \partial_{x}{ }^{\partial_{x}}}{2 \gamma}} e^{-\frac{\beta^{2}{ }^{\partial_{y} \partial_{y}}}{2 \gamma}} \Omega_{x} \Omega_{y}\right|_{x=y=0} \\
& =\left.\left\langle e^{-\frac{\alpha^{2}{ }^{x}-^{x}}{2 \gamma}}, \Omega_{x}\right\rangle_{x}\left\langle e^{-\frac{\beta^{2}{ }^{y}-y}{2 \gamma}}, \Omega_{y}\right\rangle_{y} e^{-\frac{\alpha \beta}{\gamma}-^{\partial_{x} \partial_{y}}} \Omega_{x} \Omega_{y}\right|_{x=y=0} \\
& =\exp \left(\frac{-\alpha^{2} \theta}{48 \gamma}\right) \exp \left(\frac{-\beta^{2} \theta}{48 \gamma}\right)\left\langle\Omega_{x}, \Omega_{\alpha \beta x / \gamma}\right\rangle_{x}
\end{aligned}
$$

3.5. The framed unknot and an alternative formula for $\hat{Z}^{\mathrm{LMO}}$. We now know enough to write a cleaner formula for $\hat{Z}^{\mathrm{LMO}}$. We start by computing the Kontsevich integral of the $p$-framed unknot (for an integer $p$ ). Using Lemmas 3.8 and 3.10 we get

$$
Z^{\propto}\left(\circlearrowleft^{p}\right)=\langle\Omega, \Omega\rangle^{-1} \exp \left(-\frac{p \theta}{48}\right) \exp \left(\frac{p}{2} \frown\right) \cdot \Omega \text {. }
$$

We can now compute $\AA_{0}\left(\circlearrowleft^{p}\right)$ :

$$
\begin{array}{rlrl}
\AA_{0}\left(\circlearrowleft^{p}\right) & =\langle\Omega, \Omega\rangle^{-1} \int \Omega_{x} Z^{\infty}\left(\circlearrowleft_{x}^{p}\right) d x & & \text { by Corollary } 3.12 \\
& =\langle\Omega, \Omega\rangle^{-2} \exp \left(-\frac{p \theta}{48}\right) \int \Omega_{x}^{2} \exp \left(\frac{p}{2} \overparen{x}\right) d x & \text { by Equation (19), } \\
& =\langle\Omega, \Omega\rangle^{-2} \exp \left(\frac{-p \theta}{48}\right) \exp \left(\frac{-\theta}{24 p}\right)\left\langle\Omega_{x}, \Omega_{x / p}\right\rangle_{x} & \text { by Lemma } 3.14 \\
& =\left\langle\Omega_{x}, \Omega_{x}^{-2} \Omega_{x / p}\right\rangle_{x} \exp \left[-\left(\frac{p}{48}+\frac{1}{24 p}\right) \theta\right] . &
\end{array}
$$

In particular, the normalization factors in the definition of the Århus integral are

$$
Z_{ \pm}=\AA_{0}\left(\circlearrowleft^{ \pm 1}\right)=\langle\Omega, \Omega\rangle^{-1} \exp \frac{\mp \theta}{16}
$$




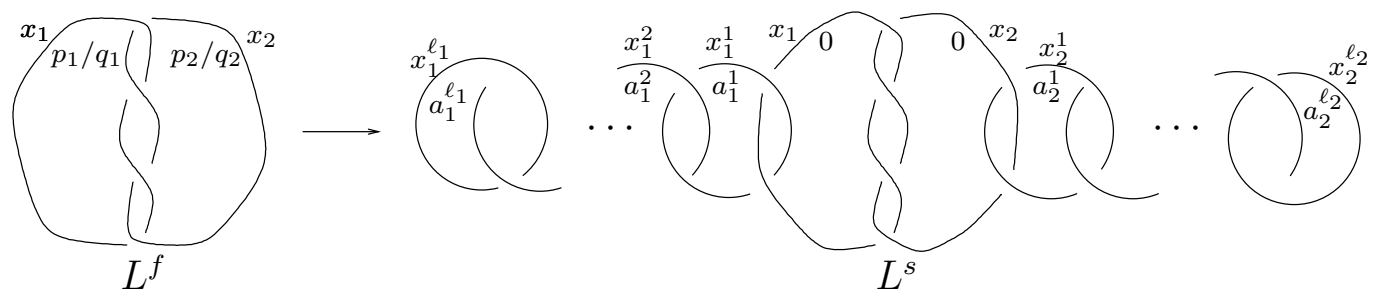

Figure 2. The link $L^{s}$ is obtained from $L$ by changing all the framings $p_{i} / q_{i}$ to 0 and shackling all the components $x_{1}, \ldots, x_{n}$ with Hopf chains marked $x_{1}^{1}, \ldots, x_{1}^{\ell_{1}}, \ldots, x_{n}^{1}, \ldots, x_{n}^{\ell_{n}}$ and framed $a_{1}^{1}, \ldots, a_{1}^{\ell_{1}}, \ldots, a_{n}^{1}, \ldots, a_{n}^{\ell_{n}}$, so that $p_{i} / q_{i}=\left\langle a_{i}^{1}, \ldots, a_{i}^{\ell}\right\rangle$ as in Equation (6).

We can now write a rather clean formula for $\hat{Z}^{\mathrm{LMO}}$ in terms of $Z^{\varnothing}$ :

Theorem 6. Let $L$ be a framed link, let $M=S_{L}^{3}$ be the result of surgery on $L$, and let $\varsigma(L)$ be the signature of its linking matrix.

- If $L$ is integrally framed, then

$$
\hat{Z}^{\mathrm{LMO}}(M)=\exp \left(\frac{\theta}{16} \varsigma(L)\right) \int Z^{\not{\alpha}}(L) \prod_{i} \Omega_{x_{i}} d x_{i} .
$$

- If $L$ is rationally framed with framing $f_{i}=p_{i} / q_{i}$ on the component $x_{i}$ (relative to the 0 framing) and if $L^{0}$ is the zero framed version of $L$, then the following surgery formula is equivalent to Theorem 1 (an in particular, as we shall later see, it holds):

$$
\hat{Z}^{\mathrm{LMO}}(M)=\exp \left(\frac{\theta}{48}\left(3 \varsigma(L)+\sum_{i} S\left(f_{i}\right)-f_{i}\right)\right) \int Z^{\not{Q}}\left(L^{0}\right) \prod_{i} e^{f_{i} \overbrace{x_{i}}{ }^{2} / 2} \Omega_{x_{i} / q_{i}} d x_{i} .
$$

Proof. The first assertion, Equation (22) is a simple assembly of Equations (3), (18) and (21). The second assertion follows by applying $\hat{\Omega}^{-1} \sigma$ to the definition of $Z(L)$ (Definition 1.6), using Wheeling and substituting the result into Equation (22).

\section{The Rational Surgery Formula}

4.1. Proof modulo computations. Let $L$ be a rationally framed link, with framing $f_{i}=$ $p_{i} / q_{i}$ on the component $x_{i}$ (measured relative to the 0 framing), and let $L^{0}$ be $L$ with all framing replaced by 0 . Let $L^{s}$ be a "shackled" version of $L$ - an integrally framed link surgery upon which is equivalent to surgery upon $L$, as in Section 2.3 and as in Figure 2. Let $X=\left\{x_{1}, \ldots, x_{n}\right\}$ be the original set of labels for the components of $L$, let $X^{e}=$ $x_{1}^{1}, \ldots, x_{1}^{\ell_{1}}, \ldots, x_{n}^{1}, \ldots, x_{n}^{\ell_{n}}$ be the set of 'extra' labels in $L^{s}$, and let $X^{s}=X \bullet X^{e}$ be the full set of labels of $L^{s}$. Let $L^{e}$ be the part of $L^{s}$ colored by $X^{e}$ (the Hopf chains). Let $M=S_{L}^{3}=S_{L^{s}}^{3}$.

In order to prove Theorem 1, we compute $\hat{Z}^{\mathrm{LMO}}(M)$ in two steps:

1. We first run the procedure of Equations (11)-(3) only on the 'extra' components of $L^{s}$. Namely, we set

$$
\check{Z}_{1}:=\nu^{\otimes X^{e}} Z\left(L^{s}\right), \quad \AA_{1}:=\int \sigma_{X^{e}} \check{Z}_{1} d X^{e}, \quad \hat{Z}_{1}^{\mathrm{LMO}}:=Z_{+}^{-\varsigma_{1+}} Z_{-}^{-\varsigma_{1}-} \AA_{1}
$$


(here $\varsigma_{1 \pm}$ are the numbers of positive/negative eigenvalues of the covariance matrix of $Z\left(L^{s}\right)$ with respect to only the variables in $\left.X^{e}\right)$.

2. We then run the procedure of Equations (11)-(B) over the remaining components, taking as our input the result of the first step. Namely, we set

$$
\check{Z}_{2}:=\nu^{\otimes X} \hat{Z}_{1}^{\mathrm{LMO}}, \quad \AA_{2}:=\int \sigma_{X} \check{Z}_{2} d X, \quad \hat{Z}_{2}^{\mathrm{LMO}}:=Z_{+}^{-\varsigma_{2}+} Z_{-}^{-\varsigma_{2}-} \AA_{2}
$$

(here $\varsigma_{1 \pm}$ are the numbers of positive/negative eigenvalues of the covariance matrix of $\left.\hat{Z}_{1}^{\mathrm{LMO}}\right)$.

Proof of Theorem 11. Theorem 11 follows immediately from Lemma 4.1 (right below) and Proposition 4.5 (on page 18), which assert that $\hat{Z}_{2}^{\mathrm{LMO}}=\hat{Z}^{\mathrm{LMO}}(M)$ and that $\hat{Z}_{1}^{\mathrm{LMO}}=Z(L)$ respectively.

Lemma 4.1. The above two step procedure really does compute $\hat{Z}^{\mathrm{LMO}}(M)$. Namely, $\hat{Z}_{2}^{\mathrm{LMO}}=$ $\hat{Z}^{\mathrm{LMO}}(M)$.

Proof. This is Theorem 6.6 of [LMO]. It also follows from the formalism of $[\AA-\mathrm{I}-\mathrm{III}]$ noting that

- Formal Gaussian integration behaves correctly under iteration ([A-II, Proposition 2.13]).

- The covariance matrix of $\hat{Z}_{1}^{\mathrm{LMO}}$ is the linking matrix of $L$ and if $\varsigma_{ \pm}$denotes the numbers of positive/negative eigenvalues of the covariance matrix of $Z\left(L^{s}\right)$, then $\varsigma_{ \pm}=\varsigma_{1 \pm}+\varsigma_{2 \pm}$.

4.2. The Hopf link and the open Hopf link. As a first step towards understanding the Kontsevich integral of shackled links, we compute $Z^{\mathscr{Q}}(H(0,0))$, the wheeled Kontsevich integral of the 0-framed Hopf link:

Proposition 4.2. The wheeled Kontsevich integral of the (x,y)-marked 0-framed Hopf link $H_{x, y}(0,0)$ is given by

$$
Z^{œ}\left(H_{x, y}(0,0)\right)=\langle\Omega, \Omega\rangle^{-1} \exp \overparen{x} .
$$

Proof. The (1,1)-framed Hopf link $H_{x, y}(1,1)$ is the $(x, y)$-marked double $\Delta_{x, y}^{t} \circlearrowleft_{t}^{1}$ of the 1-framed unknot $\circlearrowleft_{t}^{1}$, and hence

$$
\begin{aligned}
& Z^{\not}\left(H_{x, y}(1,1)\right)=\hat{\Omega}_{x}^{-1} \hat{\Omega}_{y}^{-1} \sigma_{x} \sigma_{y} Z\left(\Delta_{x, y}^{t} \circlearrowleft_{t}^{1}\right) \\
& =\hat{\Omega}_{x}^{-1} \hat{\Omega}_{y}^{-1}\left[\sigma_{t} Z\left(\circlearrowleft_{t}^{1}\right)\right]_{t \rightarrow x+y} \\
& =\hat{\Omega}_{x}^{-1}\left[\hat{\Omega}_{t}^{-1} \sigma_{t} Z\left(\circlearrowleft_{t}^{1}\right)\right]_{t \rightarrow x+y} \\
& =\hat{\Omega}_{x}^{-1}\left[Z^{\wp}\left(\circlearrowleft_{t}^{1}\right)\right]_{t \rightarrow x+y} \\
& =\langle\Omega, \Omega\rangle^{-1} \exp \left(-\frac{\theta}{48}\right) \hat{\Omega}_{x}^{-1}\left[\exp \left(\frac{1}{2} x+\overparen{y} x+y\right) \Omega_{x+y}\right] \quad \text { by (19), } \\
& =\langle\Omega, \Omega\rangle^{-1} \exp \left(-\frac{\theta}{24}\right) \exp \left(\frac{1}{2} x+\overparen{y} x+y\right) \quad \text { by Lemma 3.6, (14). }
\end{aligned}
$$




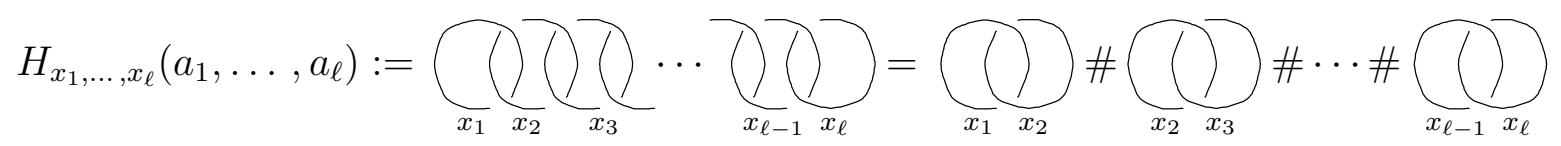

Figure 3. A Hopf chain of length $\ell$ is a connected sum of $\ell-1$ Hopf links.

It remains to undo the $(1,1)$ framing by using Lemma 3.8 on each component:

$$
\begin{aligned}
Z^{\propto}\left(H_{x, y}(0,0)\right) & =\left[\exp \left(\frac{\theta}{48}\right)\right]^{2} \exp \left(-\frac{1}{2} \overparen{x}\right) \exp \left(-\frac{1}{2} \widetilde{y} y\right) Z^{\natural}\left(H_{x, y}(1,1)\right) \\
& =\langle\Omega, \Omega\rangle^{-1} \exp \widetilde{x} .
\end{aligned}
$$

For the sake of completeness, we can now re-prove Theorem 4 :

Proof of Theorem 4 . To get $\sigma_{x} \sigma_{y} Z(\underset{x}{y})$ from $Z^{\natural}\left(H_{x, y}(0,0)\right)$, we need to apply $\hat{\Omega}_{x}$ and $\hat{\Omega}_{y}$, and to "open up" the $x$-component, which amounts to multiplication (using the product \#) by $Z\left(\circlearrowleft_{x}\right)^{-1}$ (recall that the Kontsevich integral of an open unknot is trivial). The latter operation can more easily be performed first, by $\bullet$-multiplying $Z^{\propto}\left(H_{x, y}(0,0)\right)$ by $\langle\Omega, \Omega\rangle \Omega_{x}^{-1}$, as in (17). Thus

$$
Z^{\propto}(\underset{x}{y})=\langle\Omega, \Omega\rangle \Omega_{x}^{-1} Z^{\not}\left(H_{x, y}(0,0)\right)=\Omega_{x}^{-1} \exp \widetilde{x}
$$

and hence

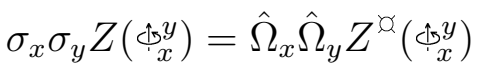

$$
\begin{aligned}
& =\hat{\Omega}_{x} \hat{\Omega}_{y}\left(\Omega_{x}^{-1} \exp \widetilde{x}\right) \quad \text { by Equation (25), } \\
& =\hat{\Omega}_{x}\left(\Omega_{x}^{-1} \hat{\Omega}_{y} \exp \widetilde{x} y\right)=\hat{\Omega}_{x}\left(\Omega_{x}^{-1} \Omega_{x} \exp \overparen{x} y\right) \\
& =\hat{\Omega}_{x}(\exp \overparen{x y})=\Omega_{y} \exp \overparen{x} \text {. }
\end{aligned}
$$

It only remains to note that $\left.\sigma_{y} Z(\underset{x}{y})=\chi_{x} \sigma_{x} \sigma_{y} Z(\underset{x}{y})=\Omega_{y} \chi_{x} \exp \overparen{x}=\Omega_{y} \exp _{\#}(\lrcorner_{x}^{y}\right)$.

4.3. The Hopf chain and the shackling element. We can use the connect-sum lemma (Lemma 3.9) repeatedly in order to compute the wheeled Kontsevich integral of Hopf chains (see Figure 3). The result is

$$
Z^{\ltimes}\left(H_{x_{1}, \ldots, x_{\ell}}(0, \ldots, 0)\right)=\langle\Omega, \Omega\rangle^{-1} \prod_{i=2}^{\ell-1} \Omega_{x_{i}}^{-1} \prod_{i=1}^{\ell-1} \exp \overbrace{x_{x_{i+1}}} .
$$

Using Lemma 3.8 and some repackaging, we get:

Proposition 4.3. Let $\left(l_{i j}\right)$ be the linking matrix of $H_{x_{1}, \ldots, x_{\ell}}\left(a_{1}, \ldots, a_{\ell}\right)$. Then

$$
Z^{\propto}\left(H_{x_{1}, \ldots, x_{\ell}}\left(a_{1}, \ldots, a_{\ell}\right)\right)=\langle\Omega, \Omega\rangle^{-1} \exp \left(-\frac{\theta \sum a_{i}}{48}\right)\left(\prod_{i=2}^{\ell-1} \Omega_{x_{i}}^{-1}\right) \exp \frac{1}{2} l_{i j} \overbrace{x_{i}} x_{j} .
$$

We can now use Proposition 4.3, Equation (25) and the connect-sum lemma (Lemma 3.9) to compute the wheeled Kontsevich integral of the shackling element (see Figure (4): 


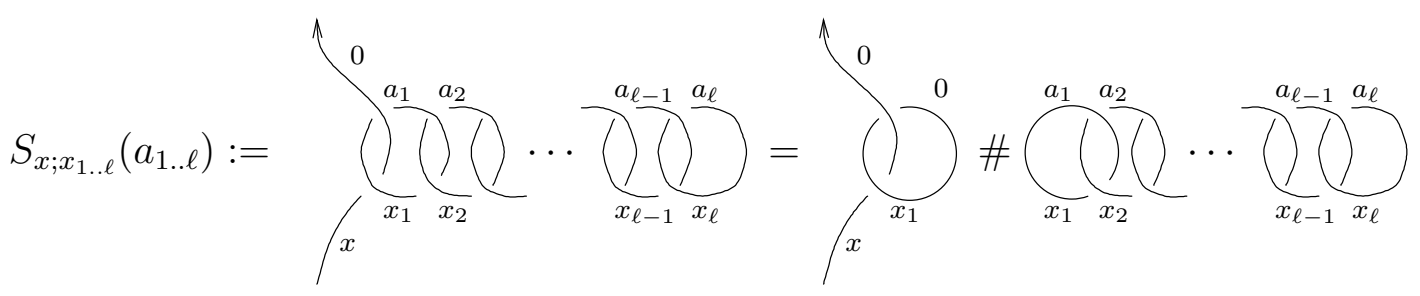

Figure 4. The shackling element is a connected sum of an open Hopf link and a Hopf chain.

Proposition 4.4. Let $\left(l_{i j}\right)$ be the $\ell \times \ell$ linking matrix of the closed components of the shackling element $S_{x ; x_{1 . . \ell}}\left(a_{1 . . \ell}\right)$. Then

$$
Z^{\not{Q}}\left(S_{x ; x_{1 . \ell \ell}}\left(a_{1 . \ell \ell}\right)\right)=\Omega_{x}^{-1}\left(\prod_{i=1}^{\ell-1} \Omega_{x_{i}}^{-1}\right) \exp (\widetilde{x}_{x_{1}}+\frac{1}{2} l_{i j} \overbrace{x_{i}} \widehat{x}_{j}-\frac{\theta \sum a_{i}}{48}) .
$$

\subsection{The Kontsevich integral of rationally framed links.}

Proposition 4.5. The output $\hat{Z}_{1}^{\mathrm{LMO}}$ of the first step of the computation procedure of Section 4.1 is equal to the Kontsevich integral for rationally framed links $Z(L)$ of Definition [1.6: $\hat{Z}_{1}^{\mathrm{LMO}}=Z(L)$.

Proof. Both sides of the required equality are clearly made of local contributions, one per each shackling element or framing fraction $p_{i} / q_{i}$. Thus it is enough to prove the proposition at the locale where all the actors act. Ergo we may as well assume that the link $L$ in question is a straight line $\uparrow_{x}^{f}$ marked $f=p / q$, and then, after choosing $\ell$ and $a_{1}, \ldots, a_{\ell}$ as in Equation (6) (more precisely, as in Equation (10)), the shackled $L$ becomes the shackling element $S_{x ; x_{1 . \ell}}\left(a_{1 . . \ell}\right)$ (so $X^{e}$, the set of "extra" labels, is $\left\{x_{1}, \ldots, x_{\ell}\right\}$ ). We just need to compute $\hat{Z}_{1}^{\mathrm{LMO}}$ as in Section 4.1, starting from $L^{s}=S_{x ; x_{1 . \ell}}\left(a_{1 . \ell \ell}\right)$. Set $C=\langle\Omega, \Omega\rangle^{-\ell} \exp \left(-\frac{\theta \sum a_{i}}{48}\right) \Omega_{x}^{-1}$ and start crunching:

$$
\begin{aligned}
& \hat{\Omega}_{x}^{-1} \sigma_{x} \AA_{1}=\langle\Omega, \Omega\rangle^{-\ell} \int Z^{\not}\left(S_{x ; x_{1 . \ell}}\left(a_{1 . . \ell}\right)\right) \prod_{i} \Omega_{x_{i}} d x_{i} \\
& =C \int \Omega_{x_{\ell}} \exp (\overparen{x_{1}}+\frac{1}{2} l_{i j} \overbrace{i}^{x_{j}}) \prod_{i} d x_{i} \\
& =C\left\langle\exp (-\frac{1}{2} l^{i j} \underbrace{x_{i} x_{j}}), \Omega_{x_{\ell}} e^{\overbrace{}^{x x_{1}}}\right\rangle_{X^{e}} \\
& =C\left\langle e^{-\frac{1}{2} l^{11} \underline{x}^{1} x_{1}} \cdot e^{-l^{1 \ell x_{1} x_{\ell}}} \cdot e^{-\frac{1}{2} l^{\ell \ell} \underbrace{x_{\ell} x_{\ell}}}, \Omega_{x_{\ell}} e^{\overbrace{}^{x x_{1}}}\right\rangle_{x_{1}, x_{\ell}} \\
& =C\left\langle e^{\frac{p}{2 q} \underline{x}^{x_{1} x_{1}}} \cdot e^{\frac{-(-1)^{\ell}}{q} x_{1} x_{\ell}} \cdot e^{\frac{v}{2 q} \underbrace{x_{\ell} x_{\ell}}}, \Omega_{x_{\ell}} e^{\overbrace{}^{x_{x}}}\right\rangle_{x_{1}, x_{\ell}} \\
& =C\left\langle e^{\frac{p}{2 q} \underbrace{x_{1} x_{1}}} \cdot e^{\frac{-(-1)^{\ell}}{q} x_{1} x_{\ell}^{x_{\ell}}}, e^{\frac{v}{2 q}{ }^{\partial_{x_{\ell}} \underbrace{\partial_{x_{\ell}}}}} \Omega_{x_{\ell}} e^{\overbrace{}^{x x_{1}}}\right\rangle_{x_{1}, x_{\ell}} \\
& =C \exp \left(\frac{v \theta}{48 q}\right)\left\langle e^{\frac{p}{2 q}{ }^{x_{1} x^{x_{1}}}} \cdot e^{\frac{-(-1)^{\ell}}{q} x^{x_{1} x_{\ell}}}, \Omega_{x_{\ell}} e^{\overbrace{x x_{1}}}\right\rangle_{x_{1}, x_{\ell}}
\end{aligned}
$$




$$
\begin{array}{rlr} 
& =C \exp \left(\frac{v \theta}{48 q}\right) \Omega_{x / q} \exp \left(\frac{p}{2 q} \widetilde{x} \hat{x}\right) & \\
& =\langle\Omega, \Omega\rangle^{-\ell} \exp \left[\left(\frac{v}{q}-\sum a_{i}\right) \frac{\theta}{48}\right] \Omega_{x}^{-1} \Omega_{x / q} \exp \left(\frac{p}{2 q} \overparen{x}\right) & \\
\hat{\Omega}^{-1} \sigma \hat{Z}_{1}^{\mathrm{LMO}} & =Z_{+}^{-\varsigma_{+}} Z_{-}^{-\varsigma} A_{1} & \\
& =\exp \left[\left(\frac{v}{q}+3 \varsigma-\tau\right) \frac{\theta}{48}\right] \Omega_{x}^{-1} \Omega_{x / q} \exp \left(\frac{p}{2 q} \overparen{x}\right) & \text { by Equation (21) } \\
& =\exp \left[(S(p / q)-p / q) \frac{\theta}{48}\right] \Omega_{x}^{-1} \Omega_{x / q} \exp \left(\frac{p}{2 q} \overparen{x}\right) & \text { by Theorem } 5 \\
\hat{Z}_{1}^{\mathrm{LMO}} & =\exp \left[(S(p / q)-p / q) \frac{\theta}{48}\right] \chi \hat{\Omega}\left(\Omega_{x}^{-1} \Omega_{x / q}\right) \exp \left(\frac{p}{2 q} \chi \hat{\Omega}(\overbrace{x})\right) & \text { by Wheeling } \\
& =\chi \hat{\Omega}\left(\Omega_{x}^{-1} \Omega_{x / q}\right) \exp \left(\frac{f}{2} \sim_{x}+\frac{S(f)}{48} \theta\right) &
\end{array}
$$

as required.

Remark 4.6. Strictly speaking, our arguments in this section assumed that $\ell \geq 2$. This is not a serious limitation. One may either read the arguments again and figure what the appropriate defaults are for $\ell<2$, or simply use a longer continued fraction expansion for $p / q$. Notice that we made no restrictive assumptions on the integers $a_{i}$, and so every fraction $p / q$ has arbitrarily long continued fraction expansions.

\section{Some Computations}

5.1. General $(p, q)$ lens spaces. As a first application of the rational surgery formula we compute the LMO invariant of general $(p, q)$ lens spaces.

Proposition 5.1. The LMO invariant of arbitrary $(p, q)$ lens spaces is given by

$$
\hat{Z}^{\mathrm{LMO}}\left(L_{p, q}\right)=\left\langle\Omega_{x}, \Omega_{x}^{-1} \Omega_{x / p}\right\rangle_{x} \exp \frac{-S(q / p)}{48} \theta
$$

Proof. Recall that the $(p, q)$ lens space $L_{p, q}$ is obtained from $S^{3}$ by surgery over the $p / q$ framed unknot $\Im^{p / q}$. Thus we can use Equation (23) to compute $\hat{Z}^{\mathrm{LMO}}$, remembering also that by Equation (17), $Z^{\propto}(\circlearrowleft)=\langle\Omega, \Omega\rangle^{-1} \Omega$ :

$$
\begin{array}{rlr}
\hat{Z}^{\mathrm{LMO}}\left(L_{p, q}\right)=\langle\Omega, \Omega\rangle^{-1} \exp \left(\frac{\theta}{48}(3 \operatorname{sign}(p / q)+S(p / q)-p / q)\right) \int e^{p \widetilde{x} / 2 q} \Omega_{x} \Omega_{x / q} d x \\
=\left\langle\Omega_{x}, \Omega_{x}^{-1} \Omega_{x / p}\right\rangle_{x} \exp \left(S\left(\frac{p}{q}\right)-\frac{p}{q}-\frac{q}{p}-\frac{1}{p q}+3 \operatorname{sign}(p q)\right) \frac{\theta}{48} & \text { by Lemma } I(1,1 / q ; p / q) \\
& =\left\langle\Omega_{x}, \Omega_{x}^{-1} \Omega_{x / p}\right\rangle_{x} \exp \frac{-S(q / p)}{48} \theta & \text { by Definition 1.5. }
\end{array}
$$

Corollary 5.2. The LMO invariant does not separate lens spaces. (Though see Section 5.4 for some more encouraging news about the LMO invariant). 
Proof. As noted in [KM, pp. 247], the lens spaces $L_{25,4}$ and $L_{25,9}$ are not homeomorphic but the Dedekind symbols $S(4 / 25)=S(9 / 25)=48 / 25$ are equal (see Table 1), and thus their LMO invariants are equal.

Exercise 5.3. Recompute $\hat{Z}^{\mathrm{LMO}}\left(L_{p, q}\right)$ directly from the Kontsevich integral of Hopf chains (Proposition 4.3), using the fact that surgery on a the Hopf chain $H_{x_{1}, \ldots, x_{\ell}}\left(a_{1}, \ldots, a_{\ell}\right)$ gives $L_{q,-p}$.

5.2. Seifert fibered spaces with a spherical base. Let $M=S^{3}\left(b ; p_{1} / q_{1}, \ldots, p_{n} / q_{n}\right)$ be the Seifert fibered space with base space $S^{2}$, with $n$ exceptional fibers with orbit invariants $\left(p_{i}, q_{i}\right)\left(p_{i} \geq 0,0<q_{i}<p_{i}, p_{i}\right.$ and $q_{i}$ relatively prime), and with bundle invariant $b \in \mathbb{Z}$. The following is easily established (see [Mon, $[\mathrm{Sd}]$ ):

- If $e_{0}:=b+\sum_{i} q_{i} / p_{i} \neq 0$, then $M$ is a rational homology sphere and $\left|H_{1}(M)\right|=\left|e_{0}\right| \prod_{i} p_{i}$.

- $M$ is given by surgery on the following (rationally framed) "key chain" link in $S^{3}$ :

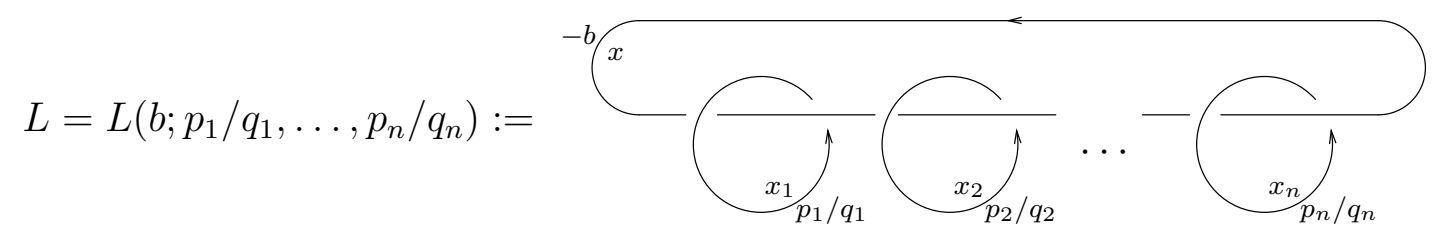

We could use the rational surgery formula of Theorem 11 to compute the LMO invariant of $M$, but it is somewhat easier to backtrack a bit, and use the main points of the proof of Theorem 1, applied in a slightly different manner. So we shackle the components $x_{1}, \ldots, x_{n}$ of $L$ as in Figure 2, and observe that the result $L^{s}$ is a $(-b)$-framed unknot marked $x$ with $n$ one-step-longer Hopf chains hanging from it. We then run the "first step" of Section 4.1 on these longer Hopf chains. As there the result is $Z^{\mathscr{Q}}\left(\circlearrowleft_{x}^{-b}\right)$ corrected by $n$ insertions of factors corresponding to the $n$ one-step-longer Hopf chains. So noting that $\left\langle 0, a_{i}^{1}, \ldots, a_{i}^{\ell}\right\rangle=$ $-1 /\left\langle a_{i}^{1}, \ldots, a_{i}^{\ell}\right\rangle$ and using Equation (26) we find that the result of the (revised) first step of the computation is

$$
\begin{aligned}
\hat{\Omega}^{-1} \sigma Z_{1}^{\prime} & =Z^{\not}\left(\circlearrowleft_{x}^{-b}\right) \prod_{i} \exp \left[\left(S\left(-q_{i} / p_{i}\right)+q_{i} / p_{i}\right) \frac{\theta}{48}\right] \Omega_{x}^{-1} \Omega_{x / p_{i}} \exp \left(-\frac{q_{i}}{2 p_{i}} \overparen{x}\right) \\
& =\langle\Omega, \Omega\rangle^{-1} \exp \left(\frac{\theta}{48}\left(e_{0}-\sum_{i} S\left(\frac{q_{i}}{p_{i}}\right)\right)\right) \Omega_{x}^{1-n}\left(\prod_{i} \Omega_{x / p_{i}}\right) \exp -\frac{e_{0} \overparen{x}}{2}
\end{aligned}
$$

This result serves as the input into the second step of the computation in the usual manner:

$$
\begin{aligned}
\hat{\Omega}^{-1} \sigma \check{Z}_{1}^{\prime} & =\langle\Omega, \Omega\rangle^{-2} \exp \left(\frac{\theta}{48}\left(e_{0}-\sum_{i} S\left(\frac{q_{i}}{p_{i}}\right)\right)\right) \Omega_{x}^{2-n}\left(\prod_{i} \Omega_{x / p_{i}}\right) \exp -\frac{e_{0} \overparen{x}}{2} \\
\check{A}_{2}^{\prime} & =\int \hat{\Omega}^{-1} \sigma \check{Z}_{1}^{\prime} d x \\
& =\langle\Omega, \Omega\rangle^{-2} \exp \left(\frac{\theta}{48}\left(e_{0}-\sum_{i} S\left(\frac{q_{i}}{p_{i}}\right)\right)\right)\left\langle\exp \frac{\underbrace{x}}{2 e_{0}}, \Omega_{x}^{2-n} \prod \Omega_{i} / p_{i}\right\rangle
\end{aligned}
$$




$$
\hat{Z}^{\mathrm{LMO}}(M)=\langle\Omega, \Omega\rangle^{-1} \exp \left(\frac{\theta}{48}\left(e_{0}-3 \operatorname{sign}\left(e_{0}\right)-\sum_{i} S\left(\frac{q_{i}}{p_{i}}\right)\right)\right)\left\langle\exp \frac{x}{2 e_{0}}, \Omega_{x}^{2-n} \prod_{i} \Omega_{x / p_{i}}\right\rangle
$$

Remark 5.4. According to [LMO, Proposition 5.3] (see also [LMMO), for any rational homology sphere $M$ the coefficient of $\theta$ in $\hat{Z}^{\mathrm{LMO}}(M)$ is $\lambda_{w}(M) / 4$, where $\lambda_{w}(M)$ denotes the Casson-Walker invariant of $M$ (see [W] in $\hat{Z}^{\mathrm{LMO}}(M)$ from Equation (27), we find that

$$
\lambda_{w}\left(S^{3}\left(b ; p_{1} / q_{1}, \ldots, p_{n} / q_{n}\right)\right)=\frac{1}{12}\left(e_{0}+\frac{2-n}{e_{0}}-3 \operatorname{sign}\left(e_{0}\right)+\sum_{i} \frac{1}{e_{0} p_{i}^{2}}-S\left(\frac{q_{i}}{p_{i}}\right)\right) .
$$

This agrees with Lescop's evaluation of the same quantity at [Les, Proposition 6.1.1] (though notice the different normalization, [Les, Section 1.5, T5.0]).

Remark 5.5. We do not know how to simplify the pairing in Equation (27) any further, except in the case when $n \leq 2$ (using Lemma 3.14). But in this case $M$ is a lens space, so we learn nothing new. At any rate, Remark 5.4 allows us to rewrite Equation (27) in an alternate way:

$$
\hat{Z}^{\mathrm{LMO}}(M)=\langle\Omega, \Omega\rangle^{-1} \exp \frac{\lambda_{w}(M) \theta}{4} \exp \left(\frac{\theta}{48 e_{0}}\left(n-2-\sum_{i} \frac{1}{p_{i}^{2}}\right)\right)\left\langle\exp \frac{\underbrace{x}}{2 e_{0}}, \Omega_{x}^{2-n} \prod_{i} \Omega_{x / p_{i}}\right\rangle
$$

Remark 5.6. Equation (29) shows that the LMO invariant of Seifert fibered spaces with a spherical base factors through at most $n+2$ integer parameters: the $p_{i}{ }^{\prime} s, e_{0}$ and the Casson-Walker invariant of $M$. As $S^{3}\left(b ; p_{1} / q_{1}, \ldots, p_{n} / q_{n}\right)$ depends on $2 n$ integers (albeit with some range restrictions), it is clear that the LMO invariant grossly fails to separate rational homology Seifert fibered spaces. Some more encouraging news about the LMO invariant are in Section 5.4.

5.3. Some $\operatorname{sl}(2)$ computations. Recall that diagram spaces such as $\mathcal{A}(\uparrow), \mathcal{A}(*)$ and $\mathcal{A}(\emptyset)$ are in some sense "universal" versions of certain tensor spaces associated with Lie algebras (see $[\mathrm{B}-\mathrm{N} \|$ ), and that the Kontsevich integral and the LMO invariant are in this sense universal versions of the Reshetikhin-Turaev invariants of links and of manifolds (see [Kas, [LM1, Le, A-1] ). Thus we wish to explicitly compare our results with some known answers for the Reshetikhin-Turaev invariants in the case of the Lie algebra $\operatorname{sl}(2)$.

In the particular case of $\operatorname{sl}(2)$, we may divide all diagram spaces $\mathcal{A}(\ldots)$ by the additional " $A_{1}$ relations" (with $\hbar$ a formal parameter),

$$
\bigcirc=3 \quad \text { and } \quad H=2 \hbar(\asymp-X) .
$$

(The first relations says that $\operatorname{sl}(2)$ is three dimensional, and the second becomes the identity $A \times(B \times C)=(A \cdot B) C-(A \cdot C) B$ in the vector calculus guise of $s l(2)$. See e.g. [CV].) Calling the corresponding quotient spaces $\mathcal{A}^{1}(\ldots)$, we easily find by repeated applications of the $A_{1}$ relations that

$$
\mathcal{A}^{1}(\emptyset)=\mathbb{Q}[[\hbar]] \quad \text { and } \quad \mathcal{A}^{1}(*)=\mathbb{Q}[[\hbar, s]],
$$


(here $s=(\frown)$ is a single strut). One easily computes that in $\mathcal{A}^{1}(\emptyset), \theta=12 \hbar$. Furthermore, simple induction (see e.g. [Th]) shows that in $\mathcal{A}^{1}(\ldots)$,

$$
\omega_{2 m}=2(2 \hbar s)^{m} \quad \text { and } \quad\left\langle s^{m}, s^{m}\right\rangle=(2 m+1) !
$$

We can now find in $\mathcal{A}^{1}(\ldots)$ :

$$
\begin{array}{rlrl}
\Omega & =\exp _{\sqcup} \sum_{m=1}^{\infty} b_{2 m} \omega_{2 m} & & \text { by Equation (4) } \\
& =\exp 2 \sum_{m=1}^{\infty} b_{2 m}(2 \hbar s)^{m} & & \text { by Equation (30) } \\
& =\frac{\sinh \sqrt{\hbar s / 2}}{\sqrt{\hbar s / 2}} & & \text { by Equation (5) } \\
& =\sum_{m=0}^{\infty} \frac{\hbar^{m}}{2^{m}(2 m+1) !} s^{m} . &
\end{array}
$$

Hence by Equation (30),

$$
\langle\Omega, \Omega\rangle=\sum_{m=0}^{\infty} \frac{\hbar^{2 m}}{2^{2 m}(2 m+1) !}=\frac{\sinh \hbar / 2}{\hbar / 2} .
$$

Assembling everything, we find from Equation (29) that in $\mathcal{A}^{1}(\emptyset)$ the LMO invariant of $M=S^{3}\left(b ; p_{1} / q_{1}, \ldots, p_{n} / q_{n}\right)$ is given as a product of an easy factor

$$
\frac{\hbar / 2}{\sinh \hbar / 2} \exp 3 \hbar \lambda_{w}(M)
$$

and a difficult 'rest':

$$
Z^{\text {rest }}(M):=\left\langle\exp \frac{s}{2 e_{0}}, \frac{2 \prod_{i} p_{i} \sinh \sqrt{\hbar s / 2 p_{i}^{2}}}{\hbar s(\sinh \sqrt{\hbar s / 2})^{n-2}}\right\rangle \exp \frac{\hbar}{4 e_{0}}\left(n-2-\sum_{i} \frac{1}{p_{i}^{2}}\right) .
$$

Next, we notice two equalities; the first is an immediate corollary of Equation (30), and the second is an exercise in Gaussian integration (for simplicity we restrict to $e_{0}>0$; otherwise we need to use a different contour for the integration):

$$
\left\langle\exp \frac{s}{2 e_{0}},\left(\frac{\hbar s}{2}\right)^{m}\right\rangle=\frac{(2 m+1) ! \hbar^{m}}{\left(4 e_{0}\right)^{m} m !}=\sqrt{\frac{\hbar^{3} e_{0}^{3}}{16 \pi}} \int_{\mathbb{R}} d \beta e^{-\hbar e_{0} \beta^{2} / 4} \beta^{2}(\hbar \beta / 2)^{2 m} .
$$

This known for every monomial in $\hbar s / 2$, we can now replace the bracket in Equation (31) by a Gaussian integral, substituting $(\hbar \beta / 2)^{2}$ for every occurrence of $\hbar s / 2$ in (31). Thus,

$$
Z^{\mathrm{rest}}(M)=\sqrt{\frac{e_{0}^{3}}{\pi \hbar}} \exp \frac{\hbar}{4 e_{0}}\left(n-2-\sum_{i} \frac{1}{p_{i}^{2}}\right) \int_{\mathbb{R}} d \beta e^{-\hbar e_{0} \beta^{2} / 4} \frac{\prod_{i} p_{i} \sinh \hbar \beta / 2 p_{i}}{(\sinh \hbar \beta / 2)^{n-2}} .
$$

Disregarding some thorny normalization issues, this result is in agreement with $\mathbb{L}$, Section 4.5]. 
5.4. The LMO invariant of integral homology Seifert fibered spaces. Corollary 5.2 and Remark 5.6 seem like bad news for finite type invariants of 3-manifolds. But things aren't as bad as they seem. The LMO invariant is a universal finite type invariant merely over the rationals, and it may well be that rational homology spheres, whose homology groups may contain torsion, are separated by finite-group-valued finite type invariants. At any rate, it is nice that we can complement our non-separation results for the LMO invariant of rational homology spheres with a separation result for (some) integral homology spheres:

Theorem 7. The LMO invariant separates integral homology Seifert fibered spaces.

Proof. To be an integral homology sphere, a Seifert fibered space must be of the form $M=$ $S^{3}\left(b ; p_{1} / q_{1}, \ldots, p_{n} / q_{n}\right)$ discussed above. Furthermore, it must have $\left|H_{1}(M)\right|=\left|e_{0}\right| \prod_{i} p_{i}=$ $\left|b+\sum_{i} q_{i} / p_{i}\right| \prod_{i} p_{i}=1$. For this to happen, $e_{0}$ must equal $\pm 1 / \prod_{i} p_{i}$, the $p_{i}$ 's must be pairwise relatively prime, and then the $q_{i}$ 's and $b$ are uniquely determined up to an overall sign by the $p_{i}$ 's, using the Chinese remainder theorem. The overall sign of $b$ and the $q_{i}$ 's (and therefore also of $e_{0}$ ) can be read from the coefficient of $\theta$ in $\hat{Z}^{\mathrm{LMO}}(M)$ (see Equation (28)). It remains to check to what extent does $Z^{\text {rest }}(M)$ determine the $p_{i}$ 's. Thus we regard $Z^{\text {rest }}(M)$ as 'known', and try to read out $n$ and $p_{1}, \ldots, p_{n}$.

For the sake of simplicity we assume that $e_{0}>0$; that is, that $e_{0}=+1 / \prod_{i} p_{i}$. This done, Equation (32) shows that $Z^{\text {rest }}(M)$ makes sense as an honest analytic function of $\hbar>0$, and not merely as a formal power series. Assume for the moment that $n>2$. For large values of $\hbar$ it is easy to bound the integral in Equation (32) above and below by rational functions of $\hbar$, and thus the growth rate of $Z^{\text {rest }}(M)$ as a function of $\hbar$ is determined by the exponential prefactor. Thus by observing the growth rate of $Z^{\text {rest }}(M)$ we can determine $\left(n-2-\sum_{i} 1 / p_{i}^{2}\right) / 4 e_{0}$, factor that term out, and therefore regard the integral in Equation (32),

$$
\sqrt{\frac{e_{0}^{3}}{\hbar}} \int_{\mathbb{R}} d \beta e^{-\hbar e_{0} \beta^{2} / 4} \frac{\prod_{i} p_{i} \sinh \hbar \beta / 2 p_{i}}{(\sinh \hbar \beta / 2)^{n-2}}=\frac{1}{\hbar^{3 / 2}} \int_{\mathbb{R}} d \beta e^{-\beta^{2} / 4 \hbar} \frac{\prod_{i} \sinh \left(\beta / 2 p_{i} \sqrt{e_{0}}\right)}{\left(\sinh \left(\beta / 2 \sqrt{e_{0}}\right)\right)^{n-2}}
$$

as known. As $\hbar$ varies, this is the integral of a fixed function against all possible Gaussians. Knowing all of these integrals we know what the function is. Thus we know all the quantities $p_{i} \sqrt{e_{0}}$ and the value of $\sqrt{e_{0}}$ (recall that the $p_{i}$ 's are all distinct and greater than 1 , so no accidental cancellations can occur). This finishes the case of $n>2$.

If $n \leq 2$, then $M$ is a sphere and therefore $\hat{Z}^{\mathrm{LMO}}(M)=1$. This leads to $Z^{\text {rest }}(M)=\frac{\sinh \hbar / 2}{\hbar / 2}$, which grows like $e^{\hbar / 2}$. This growth rate is much slower than the minimal possible value of $\left(n-2-\sum_{i} 1 / p_{i}^{2}\right) / 4 e_{0}$ for $n>2$, which is attained when $n=3$ and $\left(p_{i}\right)=(2,3,5)$. Thus the case of $n \leq 2$ is easily separated from the case of $n>2$.

Exercise 5.7. Verify directly from Equation (32) that in the $s l(2)$ case $Z^{\text {rest }}(M)=\frac{\sinh \hbar / 2}{\hbar / 2}$ for $n \leq 2$.

\section{REFERENCES}

[B-N] D. Bar-Natan, On the Vassiliev knot invariants, Topology 34 423-472 (1995).

[BGRT] _ S. Garoufalidis, L. Rozansky and D. P. Thurston, Wheels, wheeling, and the Kontsevich integral of the unknot, to appear in the Israel Jour. of Math, arXiv:q-alg/9703025.

[BLT] , T. Q. T. Le, and D. P. Thurston, in preparation.

[CV] S. V. Chmutov and A. N. Varchenko, Remarks on the Vassilliev knot invariants coming from sl ${ }_{2}$, Topology 36-1 (1997). 
[HV] V. Hinich and A. Vaintrob, Cyclic operads and algebra of chord diagrams, University of Haifa and IHES preprint, May 2000, arXiv:math.QA/0005197.

[Kas] C. Kassel, Quantum groups, Springer-Verlag GTM 155, Heidelberg 1994.

[KM] R. Kirby and P. Melvin, Dedekind sums, $\mu$-invariants and the signature cocycle, Math. Ann. 299 (1994) 231-267.

[Ko] M. Kontsevich, Deformation quantization of Poisson manifolds, I.H.E.S. preprint, September 1997, arXiv:q-alg/9709040.

[LR] R. Lawrence and L. Rozansky, Witten-Reshetikhin-Turaev invariants of Seifert manifolds, Comm. Math. Phys. 205 (1999) 287-314.

[Le] T. Q. T. Le, The LMO invariant, SUNY at Buffalo preprint, June 1999.

[LM1] and J. Murakami, The universal Vassiliev-Kontsevich invariant for framed oriented links, Compositio Math. 102 (1996), 41-64, arXiv:hep-th/9401016.

[LM2] and Parallel version of the universal Vassiliev-Kontsevich invariant, J. Pure and Appl. Algebra 121 (1997) 271-291.

[LMMO] _ - H. Murakami and T. Ohtsuki, A three-manifold invariant derived from the universal Vassiliev-Kontsevich invariant, Proc. Japan Acad. 71 Ser. A (1995) 125-127.

[LMO] _ and T. Ohtsuki, On a universal perturbative invariant of 3-manifolds, Topology 37-3 (1998) 539-574, arXiv:q-alg/9512002.

[Les] C. Lescop, Global surgery formula for the Casson-Walker invariant, Annals of Mathematics Studies 140, Princeton University Press, Princeton 1996.

[Me] N. D. Mermin, Is the moon there when nobody looks? Reality and the quantum theory, Phys. Today 39-4 (1985) 38-47.

[Moc] T. Mochizuki, On the Kirillov-Duflo type morphism, Osaka City University preprint, June 1999.

[Mon] J. M. Montesinos, Classical tessellations and three-manifolds, Springer-Verlag, 1985.

[Ro] D. Rolfsen, Knots and Links, Publish or Perish, Mathematics Lecture Series 7, Wilmington 1976.

[Sc] P. Scott, The geometries of 3-manifolds, Bull. London Math. Soc. 15 (1983) 401-487.

[Th] D. P. Thurston, Wheeling: A Diagrammatic Analogue of the Duflo Isomorphism, University of California at Berkeley Ph.D. thesis, May 2000, arXiv:math.QA/0006083.

[Wa] K. Walker, An extension of Casson's invariant, Annals of Mathematics Studies 126, Princeton University Press, Princeton 1992.

[Å-I] D. Bar-Natan, S. Garoufalidis, L. Rozansky and D. P. Thurston, The Århus integral of rational homology 3-spheres I: A highly non trivial flat connection on $S^{3}$, Selecta Math., to appear, arXiv:qalg/9706004.

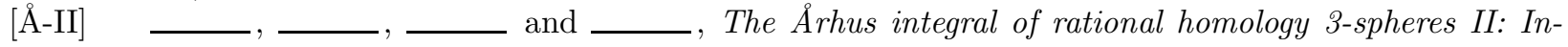
variance and Universality, Selecta Math., to appear, arXiv:math.QA/9801049.

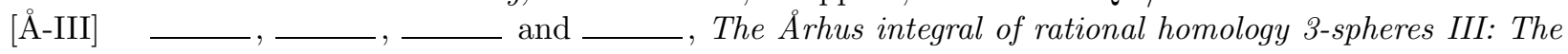
relation with the Le-Murakami-Ohtsuki invariant, Hebrew University, Harvard University, University of Illinois at Chicago and University of California at Berkeley preprint, August 1998, arXiv:math.QA/9808013.

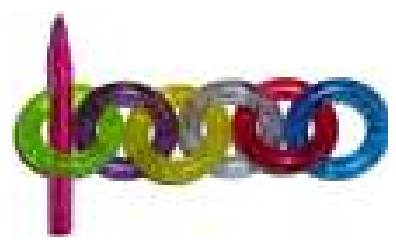

Institute of Mathematics, The Hebrew University, Giv'at-Ram, JERUSALEM 91904, ISRAEL

E-mail address: drorbn@math.huji.ac.il

URL: http://www.ma.huji.ac.il/ drorbn/

Institute of Mathematics, The Hebrew University, Giv'at-Ram, JERUSALEM 91904, ISRAEL

E-mail address: ruthel@math.huji.ac.il

URL: http://www. math.lsa.umich.edu/ ruthjl/ 